\title{
Mode I Fracture Toughness Prediction for Multiwalled-Carbon-Nanotube Reinforced Ceramics
}

\author{
Ba Nghiep Nguyen ${ }^{1}$ and Charles H. Henager, Jr.
}

Abstract

Eshelby-Mori-Tanaka models with a continuum damage mechanics approach are developed to predict the elastic damage and fracture toughness of multiwalled-carbon-nanotube (MWCNT) reinforced ceramics as a function of MWCNT fraction. This damage model is introduced in a modified boundary layer modeling approach to predict damage accumulation leading to crack propagation from a pre-existing crack tip in a process window where damage and fracture are captured under plane-strain Mode I loading. The model is validated against experimental fracture toughness data for a MWCNT 3-mol\% yttria-stabilized zirconia composite and successfully predicts the observed saturation in fracture toughness at about 25\% volume fraction MWCNTs.

\section{Keywords:}

Carbon nanotubes; Damage model; Fracture toughness; Modeling and simulation; Ceramic composites

\footnotetext{
1 Author to whom correspondence should be addressed: B.N. Nguyen, Pacific Northwest National Laboratory, PO Box 999 , MSIN: J4-55, Richland, WA 99352. Phone: (509) 375-3634; fax: (509) 375-3033; email: Ba.Nguyen@ pnnl.gov
} 


\section{Introduction and Background}

Ceramics are candidate materials for high-temperature applications, but unreinforced ceramics typically exhibit brittle behavior and are vulnerable to cracking and crack growth

22 processes leading to catastrophic failure. Therefore, without fiber or other reinforcements, ceramics are not easily used for structural applications. The science of toughening ceramics to

24 improve fracture toughness, strength, and reliability has progressed over the past decades [1-2] so that high toughness ceramic composites reinforced with strong, continuous fibers can be fabricated with toughness values greater than $20 \mathrm{MPa} \sqrt{\mathrm{m}}$ and substantial crack resistance

27 behavior (R-curves). Ceramics can also be reinforced with ductile phases, whiskers, chopped fibers, controlled debonding layers, and can be made self-reinforcing or transformation

29 toughened [2]. Each of these toughening methods can be controlled and tailored but toughness increases appear to be greatest for continuous fiber composites when the fiber/matrix interphase is designed for the proper amount of debonding during crack growth and the fibers exhibit high strength. Otherwise, toughness increases are limited [2].

With regard to toughening ceramics, there is interest in incorporating multiwalled carbon

34 nanotubes (MWCNTs), or more simply CNTs, into ceramics to produce composites with 35 improved mechanical properties for structural applications since MWCNTs possess 36 extraordinary mechanical properties that largely exceed those of conventional ceramic or carbon 37 fibers [3-4]. CNTs have been added to a wide range of materials, including polymers, metals, 38 and ceramics [4-6] and have demonstrated increases in fracture toughness and strength in certain 39 materials, mainly in polymers and brittle oxides. In brittle ceramic materials, such as zirconia [3, 40 6-8], alumina [9], silicon carbide [10], and silicon nitride [11] the increases are somewhat less

41 since synthesis of CNT-ceramic composites presents formidable challenges and enhancements in 
42 mechanical properties were not always achieved as illustrated by this summary of work in yttria 43 stabilized zirconia (YSZ), or also referred to as yttria-stabilized tetragonal zirconia polycrystal

44 (Y-TZP) [12]. The reasons for these unremarkable changes in fracture toughness were reportedly 45 due to (i) a poor dispersion of CNTs in the matrix, (ii) degradation of CNTs during processing, 46 (ii) CNT clustering at the ceramic grain boundaries, (iii) weak bonding between the CNTs and 47 the ceramic matrix, and (iv) a lower density compared to the monolithic ceramic, i.e., an 48 increased flaw population [12]. Spark plasma sintering and careful nanotube arrangements have 49 been shown to improve toughening, at least in YSZ materials, by reducing nanotube damage by 50 sintering at lower temperatures and achieving improved densification. Nanotube alignment 51 appears to play a large role relative to 3D random arrangements [13], but these arrangements are 52 difficult to achieve, and many research groups focus instead on improving dispersions of 3D 53 CNTs to avoid agglomeration and clusters of nanotubes on grain boundaries. These effects are 54 also generally observed in other material systems as noted above for $\mathrm{SiC}, \mathrm{Si}_{3} \mathrm{~N}_{4}$, and $\mathrm{Al}_{2} \mathrm{O}_{3}$ 55 matrices reinforced with CNTs.

56 Recently, Mazaheri et al. [7, 8] have processed CNT reinforced 3\%-yttria stabilized zirconia 57 (3YSZ) composites by spark plasma sintering (SPS) and have shown that reinforcing 3 YSZ with 58 CNTs results in significant increases in stiffness and fracture toughness as long as the CNT 59 contents can be homogenously distributed in the ceramic matrix. The latest results from this 60 group demonstrate excellent CNT dispersion in a 3Y-TZP matrix consolidated by SPS and a 61 measured fracture toughness $K_{\mathrm{I}}^{\mathrm{c}}$ above $15 \mathrm{MPa} \sqrt{\mathrm{m}}$ using indentation methods, which, however, 62 often give higher values compared to bulk tests. It is also not clear from this work if the 63 toughening is due to the fine grain size, from improved transformation toughening, or from CNT 
64 toughening. In any event, these CNT composites are gradually improving but many issues 65 remain to be resolved [12].

66 In the meantime, predictive models for mechanical properties (i.e., elastic properties, strength 67 and fracture toughness) of CNT ceramic composites are lacking and such models can guide 68 processing of these materials. As mentioned earlier, polymers, ceramics and metals have all been 69 used as the matrix material for producing composites with CNT reinforcements, however $70 \mathrm{CNT} /$ polymer composites have been the most studied system due to the high ultimate tensile 71 strain of polymers and the ease of processing, functionalization, tailoring, and fabrication of 72 polymer composites [14]. Mechanical property prediction models have been developed for $73 \mathrm{CNT} /$ polymer composites, but an equivalent body of models is absent in the literature for $74 \mathrm{CNT} /$ ceramic composites. In this section, we will review the key models developed for $75 \mathrm{CNT} /$ polymer and CNT/metal composites, in particular, with a focus on the Eshelby-Mori76 Tanaka (EMT) based models [15-17] as these models have been proved to be sufficiently 77 accurate and efficient to justify the development of an Eshelby-Mori-Tanka approach (EMTA) to $78 \mathrm{CNT} /$ ceramic composites developed and described in the following sections. The research and 79 challenges in modeling CNT composites have been discussed in [18].

80 Reinforcing a matrix material with CNTs can produce a composite with higher stiffness, 81 strength, and toughness than the unreinforced material. Such increased mechanical properties are 82 not solely the result of the excellent mechanical properties of CNTs but also due to an adequate 83 dispersion of CNTs in the matrix and a strong interfacial adhesion between the CNTs and matrix 84 material, which can be obtained through chemical functionalization of the CNT surface in 85 contact with the matrix material. Without this functionalization CNTs interact with the 86 surrounding matrix mainly through the Van der Waals force, which do not provide for efficient 
87 load transfer across the CNT/matrix interface [19]. Conceptually, the chemical functionalization 88 represents the CNT/matrix "interphase". Predictive models should capture this nanoscale feature 89 in order to accurately predict the increase in mechanical properties of well-processed CNT 90 composites.

91 Odegard et al. [20] were among the pioneering authors who developed a multiscale modeling 92 approach to predict the elastic properties of single-walled (SW) CNT/polymer composites 93 linking molecular dynamics (MD) to continuum modeling in conjunction with an EMT method. 94 These authors first used MD analyses to establish a model for the molecular structure of the 95 nanotube and the adjacent polymer chains [20]. Next, their equivalent-continuum model [21] was 96 applied to build the effective or equivalent fiber representing the nanotube and local polymer, 97 and finally, the EMT micromechanics combined with fiber orientation averaging were applied to compute the elastic properties of the CNT/polymer composite. Their results show substantial 99 increase in longitudinal elastic modulus with nanotube alignment and volume fraction [20]. MD 100 analyses were also used by Frankland et al. [22], but without combining with an EMT model, to 101 determine the stress/strain behavior of CNT/polymer composites. The effects of nanotube 102 waviness on the elastic modulus of CNT/polymer composites were studied by Fisher et al. [23] 103 and Bradsaw et al. [24]. These authors used finite element analyses in conjunction with an EMT 104 model and found significantly lower modulus for wavy nanotube composites than the values for 105 straight nanotube composites. Shi et al. [25] also applied an EMT model to compute the elastic 106 properties of wavy CNT/polymer composites. In their model, a wavy CNT was modeled as a 107 helical spring, and nanotube agglomeration was treated. It was found that both nanotube 108 waviness and agglomeration significantly reduce the stiffening effect of CNTs. More recently, 109 Shao et al. [26] replaced curved CNTs and those with partial debonding by equivalent straight 
110 short fibers embedded in a matrix material, and then applied a Mori-Tanaka scheme to compute

111 the effective moduli of the resulting composite. They found that both debonding and waviness

112 reduce the elastic stiffness significantly. The effect of nanotube waviness and random orientation

113 on the nanotube elastic properties and stress/strain response were also investigated by $\mathrm{Li}$ and

114 Chou [27] using a micromechanical model combined with finite element analyses. In their

115 model, a two-dimensional description of the CNT/polymer composite is achieved by discretizing

116 in finite elements a representative domain containing either aligned CNTs, or wavy CNTs, or

117 random curved CNTs. Fracture inside the domain was predicted by the maximum stress

118 criterion, and their results showed that relative to straight nanotubes, CNT waviness reduces the

119 composite elastic modulus but increases its ultimate strain while randomly oriented wavy CNTs

120 reduce both the composite elastic modulus and tensile strength.

121 Seidel and Lagoudas [28] explored EMT, self-consistent, and composite cylinder

122 micromechanics to predict the elastic properties of CNT composites. Clustering of CNTs in a 123 polymer matrix was treated using a tessellation procedure. In addition to clustering, these authors 124 also investigated the effects of $\mathrm{CNT} /$ matrix interphase regions (due to functionalization and 125 polymer wrapping) on the composite elastic properties using a multi-layer composite cylinder 126 approach that extended the Hashin and Rosen [29] model. Their model also predicted the 127 increases in both longitudinal and transverse elastic moduli for the composite as the number of 128 nanotube walls increases [28]. It was also found that randomly oriented CNT composite results 129 obtained by the EMT method compared well the experimental data [30].

130 There has been little modeling work on CNT/metal matrix composites. The most remarkable 131 work seems to be performed by Barai and Weng [31] who developed a two-scale 132 micromechanical model to study the plasticity of CNT metal matrix composites. The EMT 
133 approach was adopted to treat both large-scale and small-scale problems. The effects of CNT 134 agglomeration and interface condition on plastic strength of the composites were investigated, 135 and Barai and Weng's study showed that CNTs are a very effective agent but CNT 136 agglomeration and poor interface condition can severely reduce the composite stiffness and 137 elastoplastic strength. More recently, Dong et al. [32] developed a dislocation density based 138 model combined with EMT micromechanics to study size dependent strengthening mechanisms 139 in CNT/metal matrix composites. It was found that refinement of matrix grains does not only 140 enhance the strength of the matrix, but also weakens the thermal expansion mismatch 141 strengthening induced by the reinforcement.

142 The literature survey shows that a significant number of studies have been conducted using 143 the EMT approach to predict the elastic properties of CNT composites, in particular $144 \mathrm{CNT} /$ polymer composites, but little work was done to address the nonlinear composite behavior 145 due to damage or plasticity of the matrix material. Multiscale models accounting for progressive 146 damage are needed to predict the composite stress/strain response up to failure to predict 147 composite fracture strengths. In addition, such models combined with finite element analyses are 148 needed to predict fracture toughness of CNT composite as a function of governing 149 microstructural parameters.

150 This paper describes the development of a multiscale modeling approach to predict fracture 151 toughness of CNT-reinforced ceramic composites to provide guidance for processing these 152 materials for structural applications. Straight nanotubes are assumed, and thus the current work 153 does not account for nanotube waviness. It includes (i) prediction of elastic properties using an 154 Eshelby-Mori-Tanka approach (EMTA) [15-17], (ii) development of a mechanistic damage 155 model accounting for matrix cracking to predict the composite nonlinear stress/strain response to 
156 tensile loading to failure, and (iii) application of this damage model in a modified boundary layer 157 (MBL) analysis [33-35] using the ABAQUS ${ }^{\circledR}$ finite element package to predict fracture 158 toughness and crack resistance behavior (R-curves) for ceramic materials containing CNTs at 159 various volume fractions.

160 The modeling work provides insight and guidance to processing these materials for 161 composite microstructures to achieve optimal mechanical properties for structural applications.

162 The predictions illustrated in the following sections show that homogeneously dispersed CNTs in 163 a ceramic matrix significantly increase the resulting composite stiffness, strength, and fracture 164 toughness. However, the fracture toughness increases may not attain the levels needed for the 165 applications of these materials as structural materials, especially in nuclear reactors. In addition, 166 even assuming well-distributed CNTs, the tensile strength and fracture toughness values saturate 167 at moderate CNT volume fractions and tend to decrease at relatively high CNT volume fractions. 168 In this paper, predictions of fracture toughness as a function of CNT volume fraction have been 169 validated against the experimental data for a MWCNT nanostructured zirconia composite system 170 determined by Mazaheri et al. [8]. The results of this article are valuable guidance to the 171 experimental processing of CNT composites and suggest that the development of additional 172 toughening mechanisms needs to be explored in order to achieve the goals of adequate fracture 173 toughness for structural applications.

\section{2. Prediction of Elastic Properties}

\section{$175 \quad 2.1$ Computational Method}

176 It has been reported that distributing nanoparticles including CNTs in a matrix material (i.e., 177 polymer or ceramic) at contents for which the particles can be well-dispersed leads to substantial 178 increases in elastic moduli for the reinforced material [4, 7, 8]. However, applications of 
179 established micromechanical models do not provide accurate predictions of elastic properties if 180 the functionalization interphase formed between the nanotube and the matrix material is ignored. 181 This interphase is a nano-effect responsible for important increase in stiffness [20, 28] for the as182 formed composite compared to unreinforced ceramics or ceramics reinforced with conventional 183 fibers. Figure 1 shows a schematic of the computational procedure to compute the elastic 184 properties of a CNT composite from the properties and features of the constituents. In this work, 185 the Eshelby-Mori-Tanaka approach (EMTA) is used at each stage of the computation illustrated 186 in Figs 1a to 1c. The stiffness of a UD composite based on EMTA is given by [15-17]:

$$
\mathbf{H}=\mathbf{H}_{\mathrm{m}}+f\left(\mathbf{H}_{\mathrm{f}}-\mathbf{H}_{\mathrm{m}}\right) \mathbf{A}
$$

where $\mathbf{H}_{\mathrm{f}}$ and $\mathbf{H}_{\mathrm{m}}$ are the stiffness tensors of the inclusion and the matrix material, respectively; $f$ 189 is the inclusion volume fraction, and $\mathbf{A}$ is the inclusion strain concentration tensor given by

$$
\mathbf{A}=\mathbf{T}[(1-f) \mathbf{I}+f \mathbf{T}]^{-1}
$$

191 with

$$
\mathbf{T}=\left[\mathbf{I}+\mathbf{S} \mathbf{H}_{\mathrm{m}}^{-1}\left(\mathbf{H}_{\mathrm{f}}-\mathbf{H}_{\mathrm{m}}\right)\right]^{-1}
$$

where $\mathbf{I}$ is the identity tensor, and $\mathbf{S}$ the Eshelby tensor that depends on the inclusion aspect ratio 193 and the matrix elastic properties. Mura [36] gives expressions of $\mathbf{S}$ for different inclusion shapes. 194 The stiffness tensor of a composite with a fiber orientation distribution (Fig. 1c) is determined 195 from the solution of the UD composite (Eq. 1) that is averaged over all possible fiber orientations 196 using the Advani-Tucker model [37]:

$$
\begin{aligned}
& \bar{H}_{i j k l}=B_{1} \tilde{a}_{i j k l}+B_{2}\left(a_{i j} \delta_{k l}+a_{k l} \delta_{i j}\right)+B_{3}\left(a_{i k} \delta_{j l}+a_{i l} \delta_{j k}+a_{j l} \delta_{i k}+a_{j k} \delta_{i l}\right) \\
& \quad+B_{4} \delta_{i j} \delta_{k l}+B_{5}\left(\delta_{i k} \delta_{j l}+\delta_{i l} \delta_{j k}\right)
\end{aligned}
$$

198 where the coefficients $B_{i}(i=1,5)$ are the invariants of the stiffness tensor of the UD transversely 199 isotropic composite (Eq. 1) given in [37]. Tensors $a_{i j}$ and $\tilde{a}_{i j k l}$ are the second and fourth-order 
orientation tensors, respectively, and $\delta_{i j}$ is the identity tensor. Tensor $a_{i j}$ is either predicted by a 201 process model or measured, and then a closure approximation is used to estimate the fourth-order tensor $\tilde{a}_{i j k l}$ from $a_{i j}$. Wetzel's closure approximation [38] is used in this work.

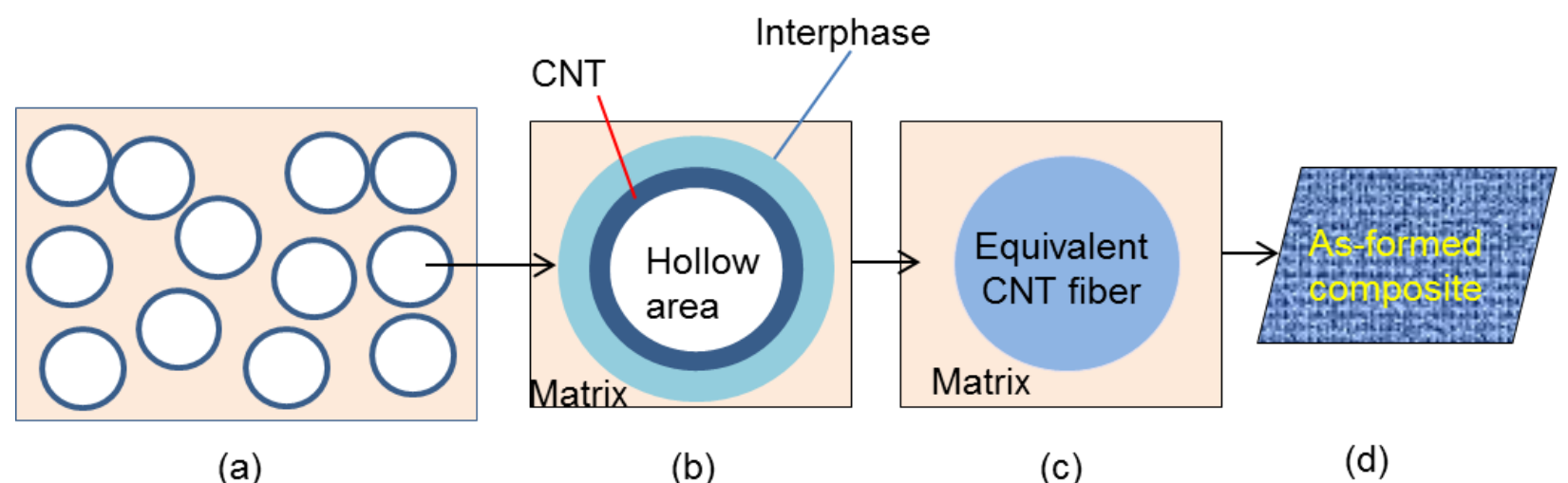

203

204

205

206

207

208

209

210

211

212

213

214

215

216

217

Fig. 1. Schematic of the EMTA homogenization procedure.

The elastic properties of a CNT composite are computed based on the procedure depicted in Fig. 1. The computation considers the UD composite containing aligned CNTs (Fig. 1a) from which the first EMTA homogenizations are performed to obtain the homogenized stiffness of the CNT/hollow space and of the CNT/interphase system (Fig. 1b). If the number of walls increases, the hollow area is reduced accordingly. Next, the second round of EMTA homogenization is carried out to determine the stiffness of the UD composite containing aligned equivalent fibers embedded in a matrix material (Fig. 1c). Finally, the fiber orientation averaging technique [37] (Eq. 4) is applied to compute the stiffness of the as-formed composite. In this paper, CNTs are assumed to be well dispersed and randomly oriented in a ceramic matrix, and as a consequence, the components of $a_{i j}$ are: $a_{11}=a_{22}=a_{33}=1 / 3$ and $a_{i j}=0$ with $i \neq j$.

For an illustration of the computational method to estimate the elastic properties of MWCNTs, the EMTA calculation considers nanotubes having a $12.5 \mathrm{~nm}$-external radius and $0.34 \mathrm{~nm}$-thick individual walls [28]. Figure 2 presents the predictions for the MWCNT elastic 
218 moduli as a function of the number of walls. It shows that both longitudinal and transverse 219 moduli increase with increasing number of walls, and for large numbers of walls, the moduli 220 achieve the elastic modulus of graphene ( $\square \approx 915 \mathrm{GPa})$. Using MWCNTs instead of SWCNTs 221 reduces the hollow space inside the tubes and consequently increases the CNT elastic moduli 222 accordingly and renders the tube behavior isotropic with the transverse elastic modulus 223 approaching the longitudinal modulus (Fig. 2) for a large number of walls. These findings agree 224 with the results reported by Seidel and Lagoudas [28] using the composite cylinder/self225 consistent method. In this paper, MWCNTs are assumed to have a large number of walls so that 226 they achieve the elastic modulus of graphene for both the longitudinal and transverse directions.

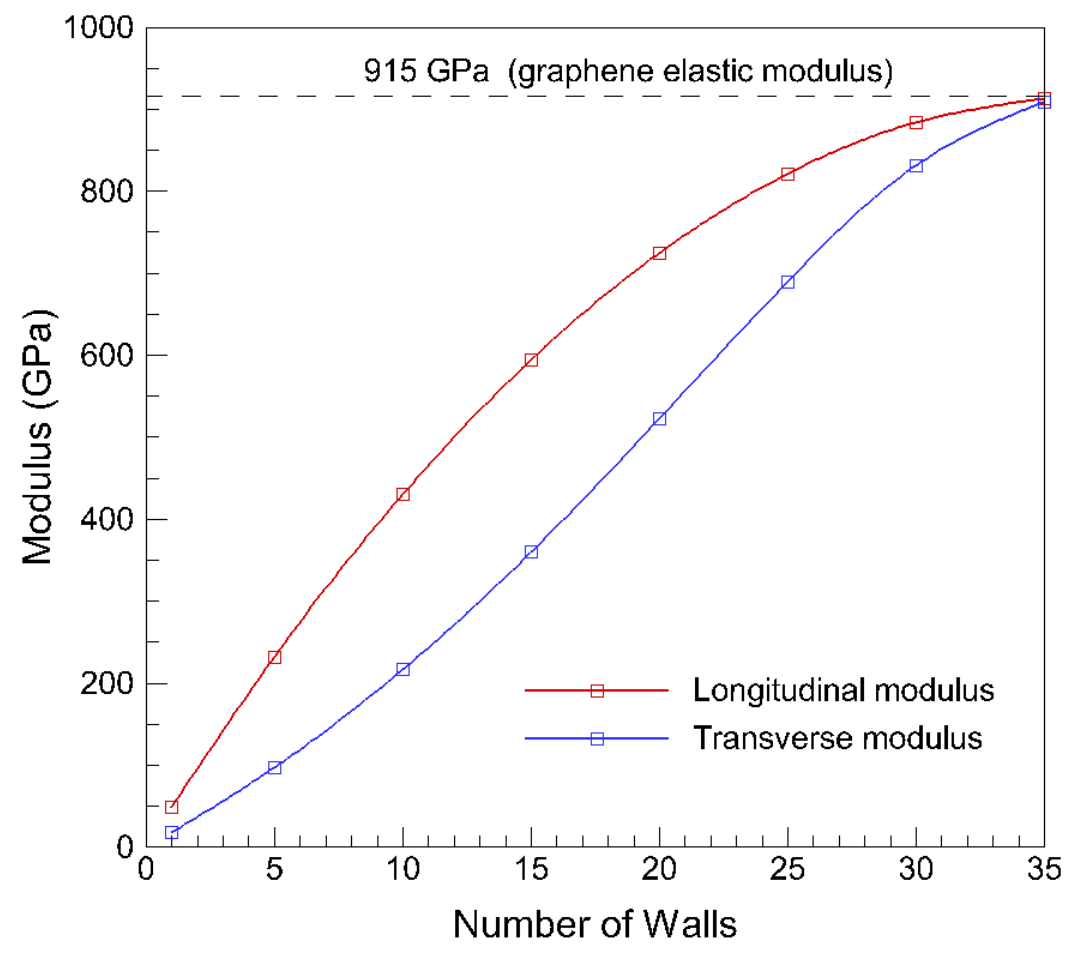

228 Fig. 2. EMTA Prediction of for the equivalent (homogenized) CNT elastic moduli as a function 229 of the number of walls. 


\subsection{Prediction of Elastic Properties for MWCNT/3 YSZ}

This section applies the EMTA method to predict the elastic properties of a MWCNT/3YSZ composite processed and studied by Mazaheri et al. [8]. The MWCNTs are about $20 \mu \mathrm{m}$ long and have a diameter about $10-20 \mathrm{~nm}$. The transmission electron microscopy (TEM) images by these authors [8] for a 5-wt\%-MWCNT/3YSZ show a homogenous grain size distribution and fairly homogeneous distributions of CNTs in the nanostructured zirconia ceramic. Also, clean interfaces between CNTs and zirconia grains were reported. However, no information about the thickness and elastic properties of the functionalized interphase was available, as this information was not obtained experimentally. In this paper, an EMTA reverse engineering method is used to identify the elastic properties of the MWCNT/3YSZ interphase. As the thickness and elastic properties of the interphase are not known, the reverse engineering procedure assumes a value for the interphase thickness (equal to half of the MWCNT radius) to solve for the elastic properties using EMTA. The procedure starts using the elastic properties of MWCNTs determined in the previous section and the measured elastic properties of the $3 \mathrm{YSZ}$ matrix [7-8], and an initial guess values for the elastic modulus and Poisson's ratio of the interphase. The selected values should be lie between the elastic properties of MWCNTs and those of the 3 YSZ matrix. The Poisson ratio for MWCNTs was determined previously from the computation of the elastic properties of MWCNTs and is 0.26. 3YSZ's Poisson ratio used in the computation is 0.27 computed from the Young's and shear moduli reported in [7-8]. If the EMTA prediction for the elastic modulus of the as-formed composite is compared with the measured value within the $2 \%$ relative error, the iterative procedure stops, and the values of the interphase elastic properties are identified. If not, adjustments of the interphase elastic properties 
252 are conducted, and the EMTA computation continues with the updated values for the interphase 253 elastic properties until the $2 \%$ accuracy agreement is reached.

254

255 256

257

258

259

260

261

262

263

264 The agreement between the predicted and measured elastic moduli for a given range of MWCNT

265 volume fractions validates the EMTA reverse engineering approach to identify the interphase 266

Fig. 3. Predicted shear modulus compared to measured data [7-8] for a MWCNT/3YSZ system as a function of MWCNT volume fraction.

The shear moduli of sintered MWCNT/3YSZ composites with MWCNT weight fractions from 0 to $5 \%$ to were experimentally determined [8]. In this paper, the measured shear modulus for $1.5 \mathrm{wt} \%$ MWCNTs (=92 GPa) is used by EMTA reverse engineering to determine the elastic properties of the interphase between MWCNTs and the zirconia matrix. The as-identified elastic properties are then used by EMTA to compute the elastic properties for all the MWCNT/3YSZ composites with different MWCNT contents, and the shear moduli results are reported in Fig. 3. elastic properties. Fig. 3 also presents Mazaheri et al.'s experimental data [8] and EMTA

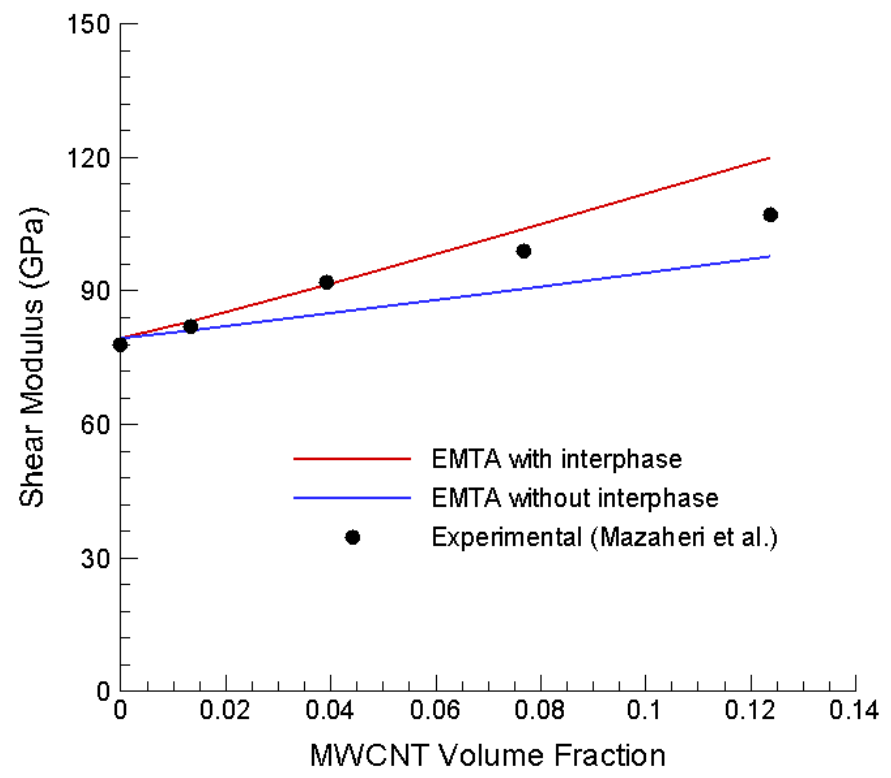


267 predictions without accounting for the functionalization interphase. It shows that the solution

268 accounting for the interphase agrees well with the experimental results while the standard

269 application of EMTA underestimates the composite shear modulus. However, we also observe

270 that the agreement between EMTA results accounting for interphase and the measured data are

271 better at low CNT volume fractions while these predictions tend to deviate from the data at

272 higher volume fractions. It is believed that the difficulty to completely disperse CNTs at high

273 concentrations in a matrix limits the enhancement of elastic modulus that could be achieved in

274 practice. In addition, increasing the volume fraction of CNTs to some extent would increase their

275 waviness and thus decrease the composite elastic modulus.

\section{3. Damage Modeling}

277 To predict the composite fracture toughness and crack resistance behavior, it is necessary to 278 model the damage development that affects the composite stiffness in order to compute the 279 composite stress/strain response up to failure. It has been reported that CNT ceramic composites 280 subjected to loadings undergo matrix cracking [39]. For composites that are well processed, the 281 functionalization interphases between CNTs and the ceramic matrix possess a strong bond that 282 prevents the debonding of nanotubes in the way that is observed for conventional fiber 283 composites. If debonding occurs, this mechanism happens inside the matrix [39]. Based on these 284 experimental observations, this paper describes matrix cracking as the key progressive 285 mechanism responsible for damage leading to failure of CNT-reinforced ceramic composites.

\subsection{Model Formulation}

287 A mechanistic damage model to describe matrix cracking in CNT ceramic composites is 288 derived from a model for glass-ceramics developed by Nguyen et al. [35] that is termed 
289 "mechanistic" since its formulation uses both micromechanics and continuum damage 290 mechanics. It assumes elastic CNTs completely randomly dispersed in a ceramic matrix whose 291 cracking behavior is described by a continuum damage description. An incremental EMTA 292 method combined with fiber orientation averaging is used to incrementally determine the 293 composite response.

294 Using a scalar damage variable, $D$, for matrix cracking, the elastic deformation energy 295 density chosen as the thermodynamic potential is expressed in terms of matrix strains, $\varepsilon_{i j}^{\mathrm{m}}$ and $D$ :

$$
\Phi\left(\varepsilon_{i j}^{\mathrm{m}}, D\right)=\frac{1}{2} H_{i j k l}^{\mathrm{m}}(D) \varepsilon_{i j}^{\mathrm{m}} \varepsilon_{k l}^{\mathrm{m}}
$$

297 where $H_{i j k l}^{\mathrm{m}}(D)$ denotes the stiffness tensor of the matrix material affected by damage 298 characterized by $D$. From Eq. 5, the matrix constitutive response and thermodynamic force 299 associated with $D$ are obtained as [40]:

$$
\sigma_{i j}^{\mathrm{m}}=\frac{\partial \Phi}{\partial \varepsilon_{i j}^{\mathrm{m}}}=H_{i j k l}^{\mathrm{m}}(D) \varepsilon_{k l}^{\mathrm{m}}
$$

301 and

$$
F=\frac{\partial \Phi}{\partial D}=\frac{1}{2} \frac{\partial H_{i j k l}^{\mathrm{m}}(D)}{\partial D} \varepsilon_{i j}^{\mathrm{m}} \varepsilon_{k l}^{\mathrm{m}}
$$

303 The Clausius-Duhem inequality, which is the dissipation criterion, reads:

$$
-F \cdot \dot{D} \geq 0
$$

If $F<0 \rightarrow \dot{D}>0$ : damage progresses

If $F \geq 0 \rightarrow \dot{D}=0$ : damage is stable

305 Next, in order to define a damage criterion, we utilize the concept of damage threshold 306 function similar to that used by Renard et al. [41] for polymer composites: 


$$
f(D)=F_{\mathrm{c}}(D)-F
$$

308 where $F_{\mathrm{c}}(D)$ is the damage threshold function of $D . F_{\mathrm{c}}(D)$ can be computed numerically using 309 Eq. 7 and the matrix stress-strain response identified from the composite stress-strain data by a 310 reverse engineering method.

311 Finally, the damage evolution law for the matrix material is obtained using the damage 312 criterion (Eq. 9) and the consistency conditions, $f=0$ and $\mathrm{d} f=0$ :

$$
\mathrm{d} D=\frac{-\frac{\partial H_{i j k l}^{\mathrm{m}}(D)}{\partial D} \varepsilon_{i j}^{\mathrm{m}} \mathrm{d} \varepsilon_{k l}^{\mathrm{m}}}{\frac{1}{2} \frac{\partial^{2} H_{i j k l}^{\mathrm{m}}(D)}{\partial D} \varepsilon_{i j}^{\mathrm{m}} \varepsilon_{k l}^{\mathrm{m}}-\frac{\partial F_{\mathrm{c}}(D)}{\partial \mathrm{D}}}
$$

314 In this paper, a linear reduction of the matrix elastic modulus with the damage variable is used $315[35,42]:$

$$
E_{\mathrm{m}}(D)=E_{\mathrm{m}}^{0}(1-D)
$$

317 where $E_{\mathrm{m}}^{0}$ is the initial elastic modulus in the undamaged state. Introducing Eq. 11 in Eq. 10, the 318 matrix damage evolution law becomes:

$$
\mathrm{d} D=\frac{\frac{\partial H_{i j k l}^{\mathrm{m}}(D)}{\partial D} \varepsilon_{i j}^{\mathrm{m}} \mathrm{d} \varepsilon_{k l}^{\mathrm{m}}}{\frac{\partial F_{\mathrm{c}}(D)}{\partial \mathrm{D}}}
$$

Eq. 12 determines the damage variable increment caused by an increment of the matrix strain $321 \mathrm{~d} \varepsilon_{i j}^{\mathrm{m}}$ due to composite deformation.

322 The composite stress-strain response is nonlinear due to matrix cracking and is given by:

$$
\sigma_{i j}=\bar{H}_{i j k l}(D) \varepsilon_{k l}
$$


324 where the composite stiffness matrix $\bar{H}_{i j k l}(D)$ is affected by matrix damage given by Eq. 4 and 325 computed using Eq. 11 in the EMTA computation procedure.

326 The matrix strain increment is computed from the composite strain increment as:

$$
\mathrm{d} \boldsymbol{\varepsilon}_{\mathrm{m}}=\mathbf{A}_{\mathrm{m}} \mathrm{d} \boldsymbol{\varepsilon}
$$

328 329 330 331 332 333

with $\mathbf{A}_{\mathrm{m}}$ being the matrix strain concentration tensor that is defined in terms of the averaged 'fiber' strain concentration tensor $\overline{\mathbf{A}}$ as:

$$
(1-f) \mathbf{A}_{\mathrm{m}}+f \overline{\mathbf{A}}=\mathbf{I}
$$

where $\mathbf{I}$ is the identity tensor, and $\overline{\mathbf{A}}$ is obtained by application of the fiber orientation averaging method [37] to the strain concentration tensor A (Eq. 2).

As the composite damage response is nonlinear, the computation is carried out incrementally. The calculation of the matrix strain increment (Eq. 14) allows computation of the damage variable increment according to the damage evolution law to update the damage variable. The matrix elastic modulus is then updated using Eq. 11 to compute the stiffness tensor for the composite by application of EMTA and fiber orientation averaging. Damage by matrix cracking can evolve according to Eqs. 8 and 12 until a critical or saturation state at which $D=D_{\text {cr }}$ (the saturation value of $D$ ), and the composite can no longer carry load and will fail. Failure of the composite leading to fiber pullout, initiation and propagation of a macroscopic crack is captured by the Nguyen et al.'s failure model [35] associated with a vanishing finite element method $[35,43]$. It is noted that fiber pullout was not modeled discretely in this work, but this damage mechanism that corresponds to the final stage of damage was captured by the failure model [35] and vanishing element method $[35,43]$ that numerically reduces the stresses and stiffness at the material failure location to zero in a number of load steps. 


\subsection{Prediction of Stress-Strain Response for MWCNT/3 YSZ}

The damage model has been introduced in EMTA-NLA (Eshelby-Mori-Tanaka Approach to Non-Linear Analysis), a Pacific Northwest National Laboratory's capability that is implemented in the $\mathrm{ABAQUS}{ }^{\circledR}$ finite element package via user subroutines for nonlinear structural analyses. This section illustrates an application of the damage model to predict the stress/strain response of the MWCNT/3YSZ composite as a function of the MWCNT volume fraction. This composite system was examined by Mazaheri et al. [8] and is modeled in Section II of this paper for elastic properties. As the experimental stress/strain data including strength are not available for these composites and the $3 \mathrm{YSZ}$ matrix, literature data and adequate assumptions must be adopted regarding the 3 YSZ matrix stress-strain behavior to be used in the model to predict the response of the MWCNT/3YSZ composite as a function of the MWCNT volume fraction. The modeling work here aims at elucidating the composite behavior to assess the levels of strength and failure strain that can be achieved under ideal conditions of complete dispersion of MWCNTs in the 3YSZ matrix and perfect bonding between the MWCNTs and matrix.

Figure 4 presents the uniaxial stress-strain curve assumed for the $3 \mathrm{YSZ}$ matrix. This curve is built considering the following data for 3YSZ: (i) Initial elastic modulus [7], $E_{\mathrm{m}}^{0}=198.12 \mathrm{GPa}$, (ii) $713 \mathrm{MPa}$ strength [44], and (iii) $0.45 \%$ failure strain. As a result, the saturation value of the damage variable is $D_{\mathrm{cr}}=1-E_{\mathrm{m}}\left(\bar{\varepsilon}_{\mathrm{m}}=0.0045\right) / E_{\mathrm{m}}^{0}=0.2$. The initial elastic modulus of $3 \mathrm{YSZ}$ measured by Mazaheri et al. [7] agrees with the experimental data by Kondoh et al. [44]. Together with the measured shear modulus [8], the 3YSZ Poisson's ratio is determined to be 0.27. The value of the failure strain adopted is justified as follows. If the behavior of the $3 \mathrm{YSZ}$ matrix were linear elastic up to rupture (at the failure stress of $713 \mathrm{MPa}$ ), the failure strain would be $0.36 \%$. However, as progressive matrix cracking develops in the composite under loading, the 
matrix stress/strain response is nonlinear up to failure, which corresponds to a failure strain

370 greater than $0.36 \%$. The adopted value of failure strain $(0.45 \%)$ on the 3 YSZ stress/strain curve

371 and all the other material parameters will be assessed in the next section where the damage

372 model is used with the MBL analysis to predict fracture toughness of MWCNT/3YSZ

373 composites.

3YSZ Matrix

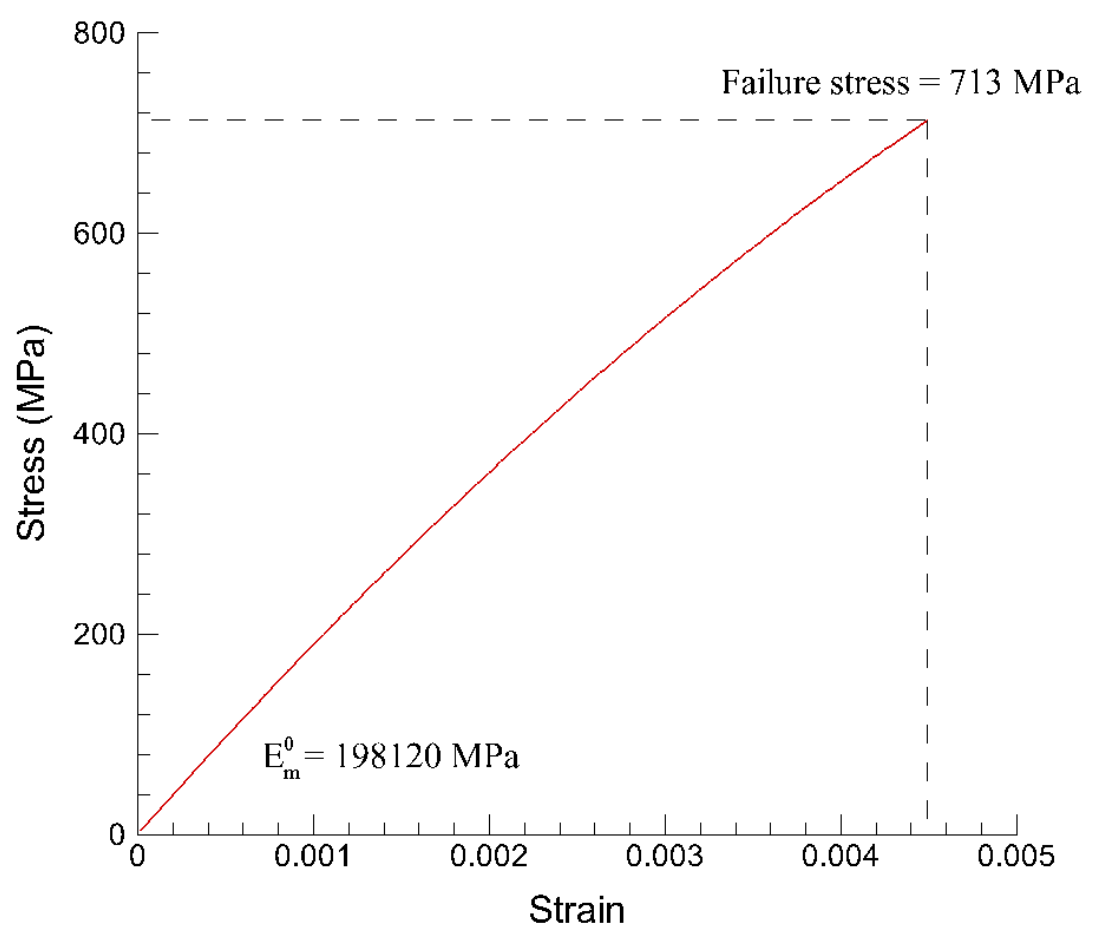

374

Fig. 4. The uniaxial stress-strain curve established for the 3YSZ matrix.

Using the matrix stress/strain response, the damage threshold function is computed in the

377 following manner. For a current value of the matrix strain, the current matrix stress and elastic

378 modulus are determined from this stress-strain curve (Fig. 4). Next, the current values of the

379 damage variable and thermodynamic force are computed using Eqs. 11 and 7, respectively. The

380 damage threshold function is obtained as: $F_{\mathrm{c}}(D)=F(D)$. In this manner, the damage threshold

381 function is computed numerically for any value of the damage variable in the $\left[0, D_{\text {cr }}\right]$ interval. 
382 Fig. 5 provides the damage threshold function computed for the 3 YSZ matrix material based on 383 the 3YSZ stress-strain curve given in Fig. 4. The as-determined values are well fitted by a 384 polynomial as illustrated on Fig. 4 that uses a second-degree polynomial.

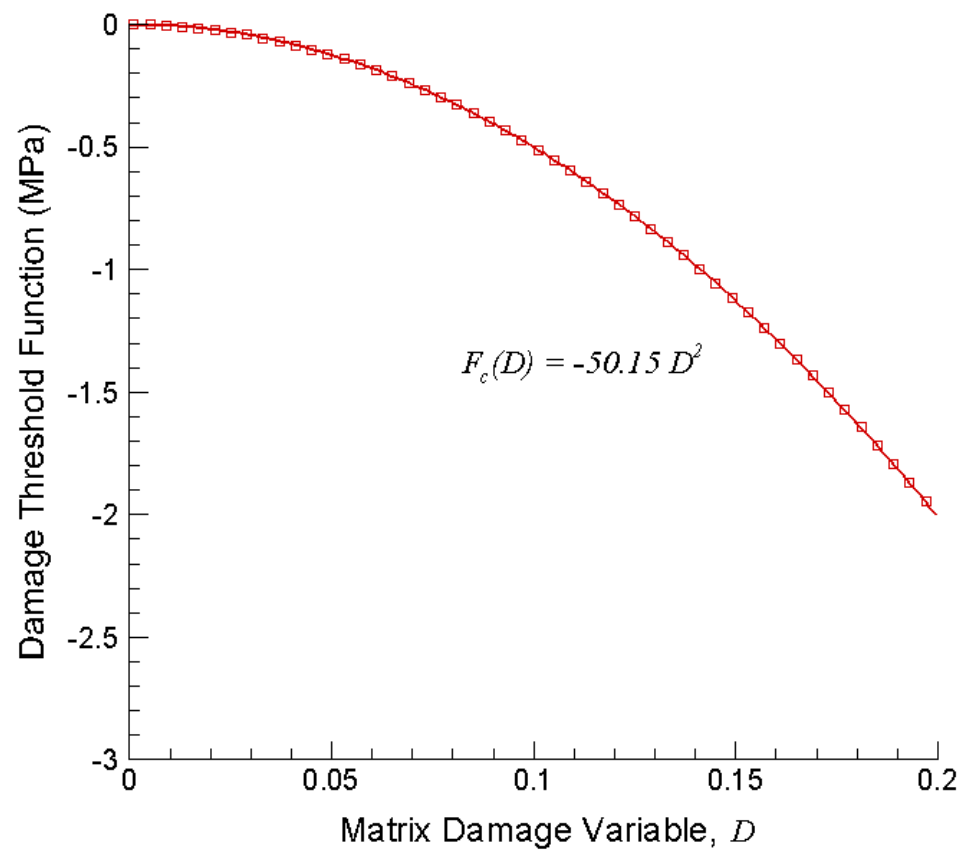

385

Fig. 5. The damage threshold function computed for the 3YSZ matrix.

The damage threshold function, the critical value of the damage variable, and the elastic properties of the 3YSZ matrix and MWCNTs are introduced as input data to EMTA-NLA for analyses of MWCNT/3YSZ composites subjected to tensile loading to failure. The computed stress-strain response of MWCNT/3YSZ as a function of the MWCNT volume fraction is reported in Fig. 6 and shows a strong effect of the MWCNT content on the composite behavior. Due to the complete fiber dispersion and perfect bonding assumptions, the initial slope of the curve (elastic modulus) increases with increasing MWCNT volume fraction. In the low to moderate MWCNT volume fraction range (less than $12.5 \%$ ), the composite strength increases significantly with increasing MWCNT volume fraction, and the strain at composite failure is 
396 little affected. However, between $12.5 \%$ and $23 \%$ volume fractions, the composite behavior

397 becomes more brittle and exhibits a significant reduction of failure strain although the composite

398 strength increases substantially. Beyond $23 \%$ but less than $32 \%$ volume fraction, the composite

399 strength saturates and computed failure strains continue to decrease, and after $32 \%$ both

400 composite strength and failure strain drop substantially. These results show that even at the ideal

401 condition of complete dispersion of MWCNTs, the strength saturates about 23\% MWCNT

402 volume fraction, and both strength and failure strain degrade substantially at higher volume

403 fractions. In practice, the difficulty in dispersing MWCNTs in a ceramic matrix material would

404 likely cause the composite strength to saturate at lower levels and at lower MWCNT volume

405 fractions than $23 \%$. We consider this value to be near ideal behavior.

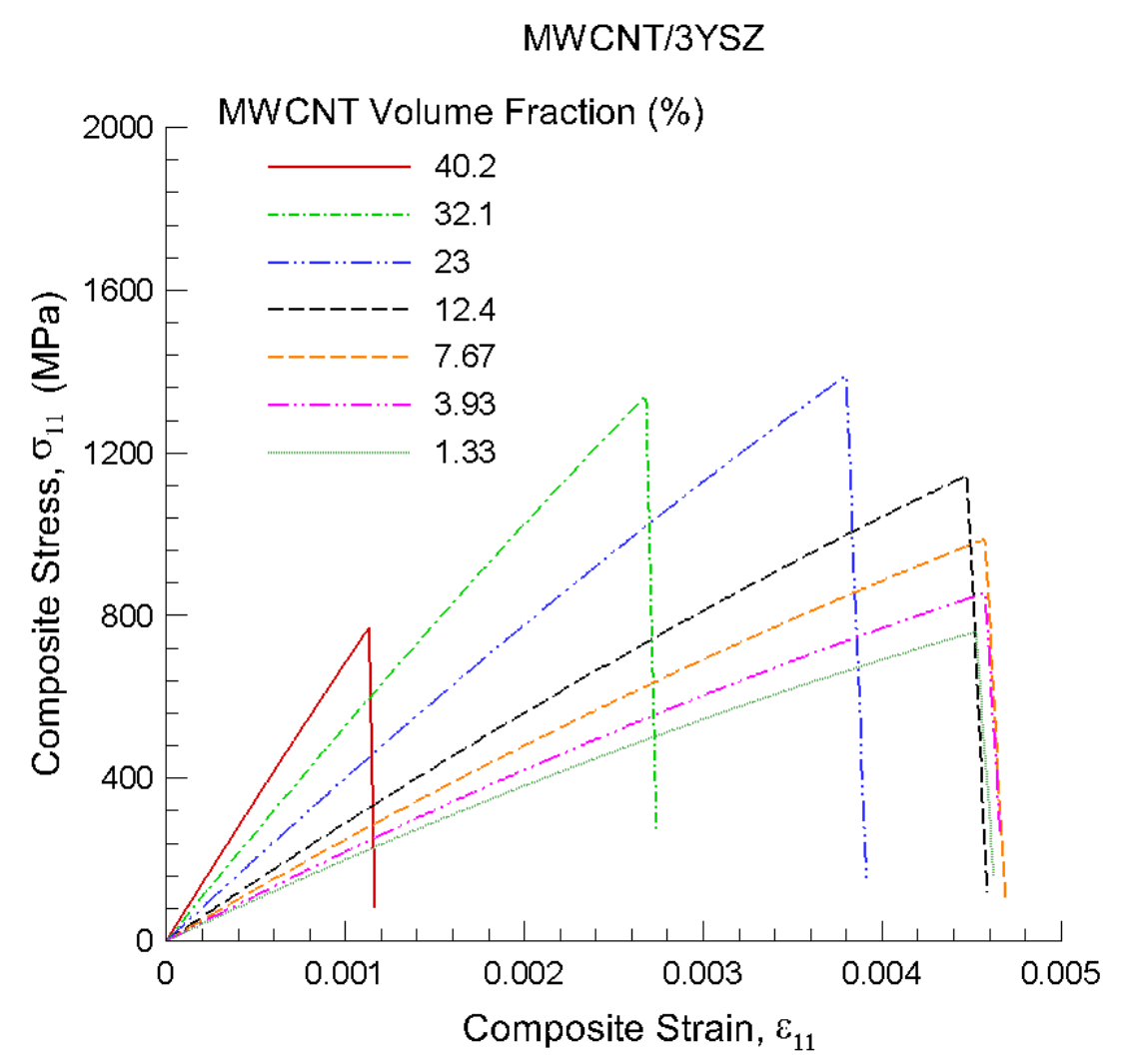

Fig. 6. Stress-strain responses predicted for the MWCNT/3YSZ composite as a function of

MWCNT volume fraction. 


\section{Fracture Toughness Prediction}

410 The damage model developed for MWCNT reinforced ceramics is used in a modified 411 boundary layer (MBL) modeling approach to predict the composite fracture toughness under

412 Mode I loading. The MBL modeling approach was initially proposed by Tvergaard and 413 Hutchinson [33] to investigate crack growth resistance in elastic-plastic solids. Later, it was also 414 used by other authors $[34,35,45]$ to study damage and fracture in a small process zone at the tip 415 of an existing crack. The principle of the MBL modeling approach is described in Fig. 7. 416 Considering an initial crack inside a solid subjected to loading at the solid boundaries (Fig. 7a).

417 If one is interested in conditions for the onset of crack propagation and growth, and the 418 governing parameters, an approach to damage and fracture at the microstructural scale is 419 appropriate. This approach is defined as small-scale damage and fracture analysis. It relies on the 420 assumption that damage and fracture is only allowed to develop in a process zone containing an 421 initial crack tip. The process zone must be much smaller than all relevant specimen/structure 422 dimensions so that a boundary layer problem can be formulated with the crack-tip fields in an 423 undamaged solid as the remote boundary conditions applied on the outer contour of a circular 424 region containing the process zone (Fig. 7b). The MBL analysis can be efficiently used to 425 characterize the material fracture toughness and crack resistance behavior without modeling the 426 whole structure with the associated boundary conditions.

427 For the CNT ceramic composites studied in this paper, the CNTs are assumed to be 428 completely randomly distributed in the ceramic matrix; thus, the homogenized behavior is 429 isotropic, and the crack-tip fields for a crack in an elastic isotropic solid are then used as the 430 remote boundary conditions for the MBL analysis. Under plane-strain Mode I loading condition, 431 the crack-tip displacement components in an elastic solid are given by: 


$$
\begin{aligned}
& u_{1}=\frac{K_{\mathrm{I}}}{2 \mu} \sqrt{\frac{r}{2 \pi}} \cos \left(\frac{\theta}{2}\right)\left[\kappa-1+2 \sin ^{2}\left(\frac{\theta}{2}\right)\right], \\
& u_{2}=\frac{K_{\mathrm{I}}}{2 \mu} \sqrt{\frac{r}{2 \pi}} \sin \left(\frac{\theta}{2}\right)\left[\kappa+1-2 \cos ^{2}\left(\frac{\theta}{2}\right)\right]
\end{aligned}
$$

where $r$ and $\theta$ are the polar coordinates centered at the crack tip. $K_{\mathrm{I}}$ denotes the Mode I loading

434 stress intensity factor, $\mu$ is the shear modulus, and $\kappa$ is given by $\kappa=3-4 v$ with $v$ being 435 Poisson's ratio.

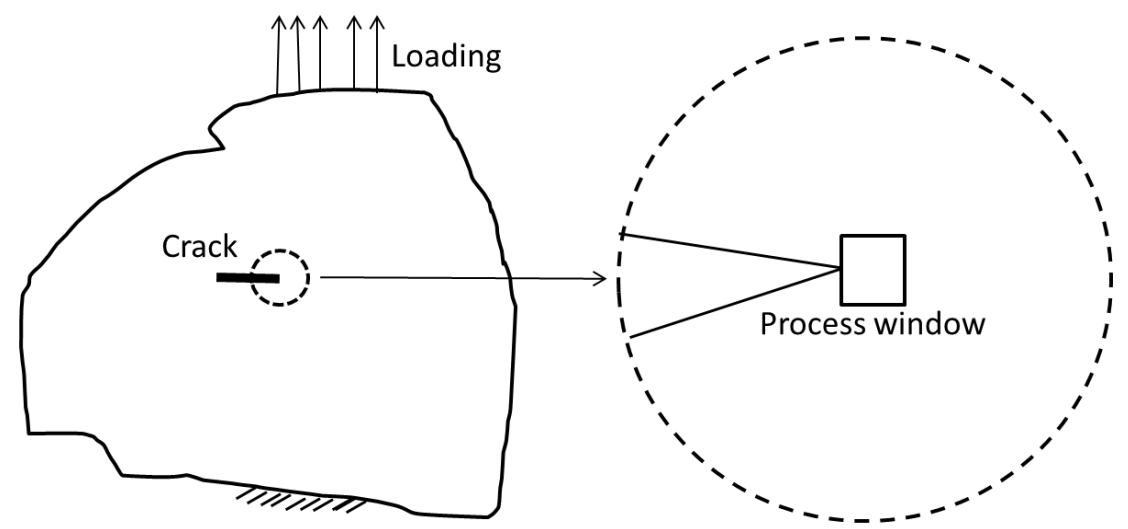

436

437 Fig. 7. Principle of the MBL modeling approach: (a) a crack inside a body is subjected to remote

438 loading, and (b) a damage process window containing the crack-tip is surrounded by a circular region where the asymptotic crack-tip fields are prescribed as boundary conditions.

The damage model developed for CNT ceramic composites discussed in the previous section 442 is used to describe the development of damage leading to crack growth in the process zone. The 443 composite behavior away from the process zone remains linear elastic. Under increasing load 444 applied via boundary conditions (Eq. 16), damage evolves at the crack tip until attaining a 445 critical stage characterized by the value of the damage variable reaching $D_{c r}$. The critical damage 446 stage represents the onset of crack propagation in the damage process window, and the 
447 corresponding value of $K_{\mathrm{I}}$ at this stage defines the material Mode I fracture toughness, $K_{\mathrm{I}}^{\mathrm{c}}$.

448 Crack propagation in the process window is captured by the vanishing element model [35].

449 Figure 8 presents the finite element mesh designed for the MBL analysis to predict fracture

450 toughness of the MWCNT/3YSZ composite system studied in this paper. The whole mesh for

451 the MBL problem is given in Fig. 8a while Fig. 8b shows a snapshot of the mesh in the process

452 window. The outer diameter of the circular region must be much greater than the size of the

453 process window so that damage and fracture occurring in the process window do not affect the

454 remote fields. In this work, the dimension of the process window and the outer diameter of the

455 circular region are $0.7 \mathrm{~mm}$ and $70 \mathrm{~mm}$, respectively. According to [8], the length of CNTs in the

456 studied composite is about 20 microns. The mesh size of the elements in the process window

457 containing the crack tip is 35 microns. This mesh size design captures the length scale for this

458 composite for continuum modeling: each element in the process window represents the 459 composite representative volume element that is formed by CNTs of the given length randomly 460 and uniformly dispersed in the 3YSZ matrix. 


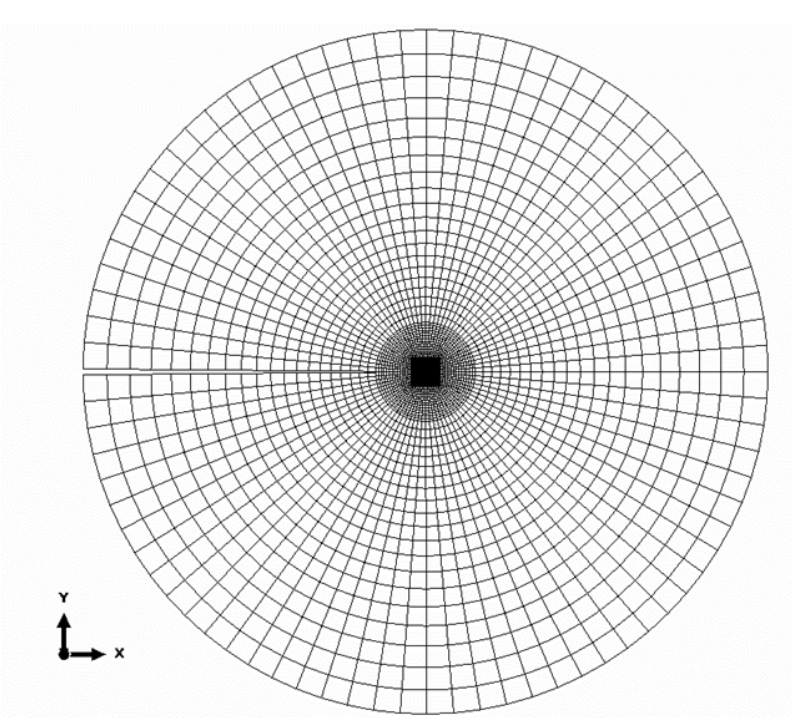

(a)

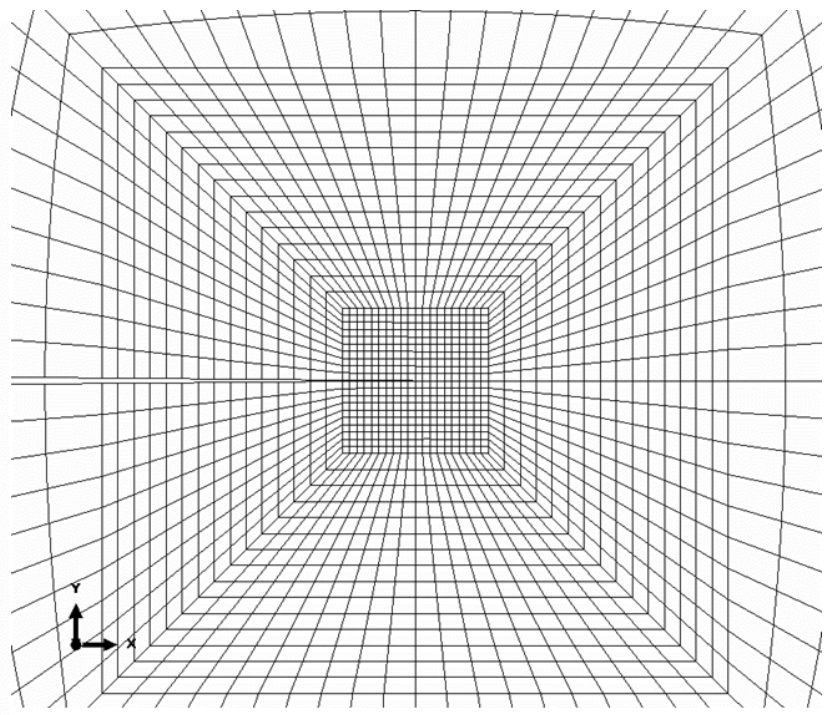

(b)
462

463

464

465

466

467

468

469

470

471 472 boundary layer problem is not achieved.

473

474

475

476 mesh for the damage/fracture process window.

It is noted that the pure Mode-I loading and the uniform material properties considered in the

MBL analysis provides the symmetry for the boundary layer problem and suggests that it would be sufficient to analyze a half of the circular region (Fig. 8(a)). However, for the sake of generality, in this paper we have chosen to model the whole circular region. Analyzing the whole region will allow us to later extend the model to incorporate randomness in material parameters and/or irregular mesh sizes and shapes in the process window for which the symmetry of the

Figure 9 illustrates the contour of the damage variable in the process window at the onset of crack propagation predicted by the damage model for the composite with 3-wt\% MWCNTs (0.077 volume fraction). The corresponding value of the stress intensity factor, $K_{\mathrm{I}}$, inducing this damage distribution is $7.78 \mathrm{MPa} \sqrt{ }$. Fig. 9 shows that the damage variable has attained the 
477 critical value $\left(D_{\text {cr }}=0.2\right)$ at the crack tip for this loading level, and the stress intensity factor is 478 then identified as the critical stress intensity factor or fracture toughness $479 \quad\left(K_{\mathrm{I}}=K_{\mathrm{I}}^{\mathrm{c}}=7.78 \mathrm{MPa} \cdot \sqrt{\mathrm{m}}\right)$.

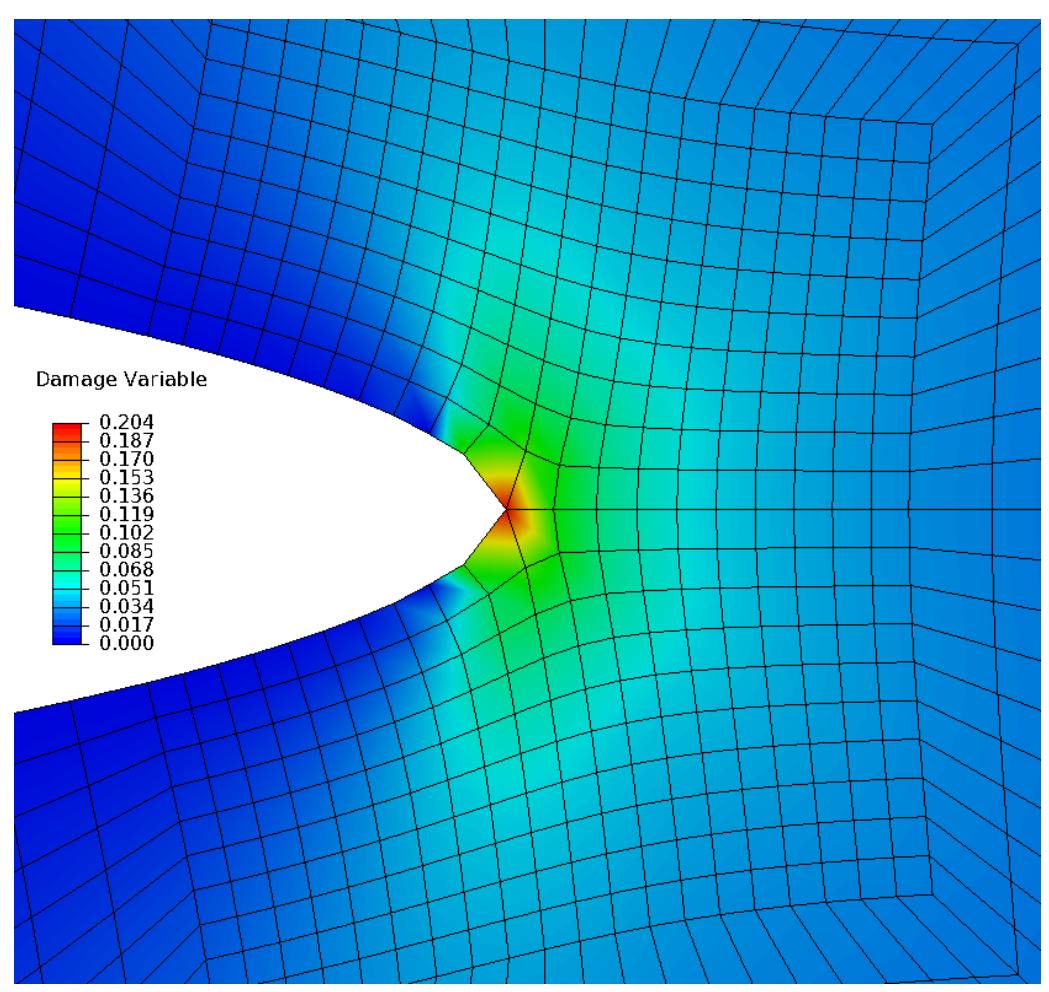

Fig. 9. Predicted damage distribution at the onset of crack propagation for the $3-\mathrm{wt} \%$ MWCNT/3YSZ. 
491 also given in Fig. 10. The agreement between the predicted and experimental results validates the 492 material parameters identified for the damage model in the previous section.

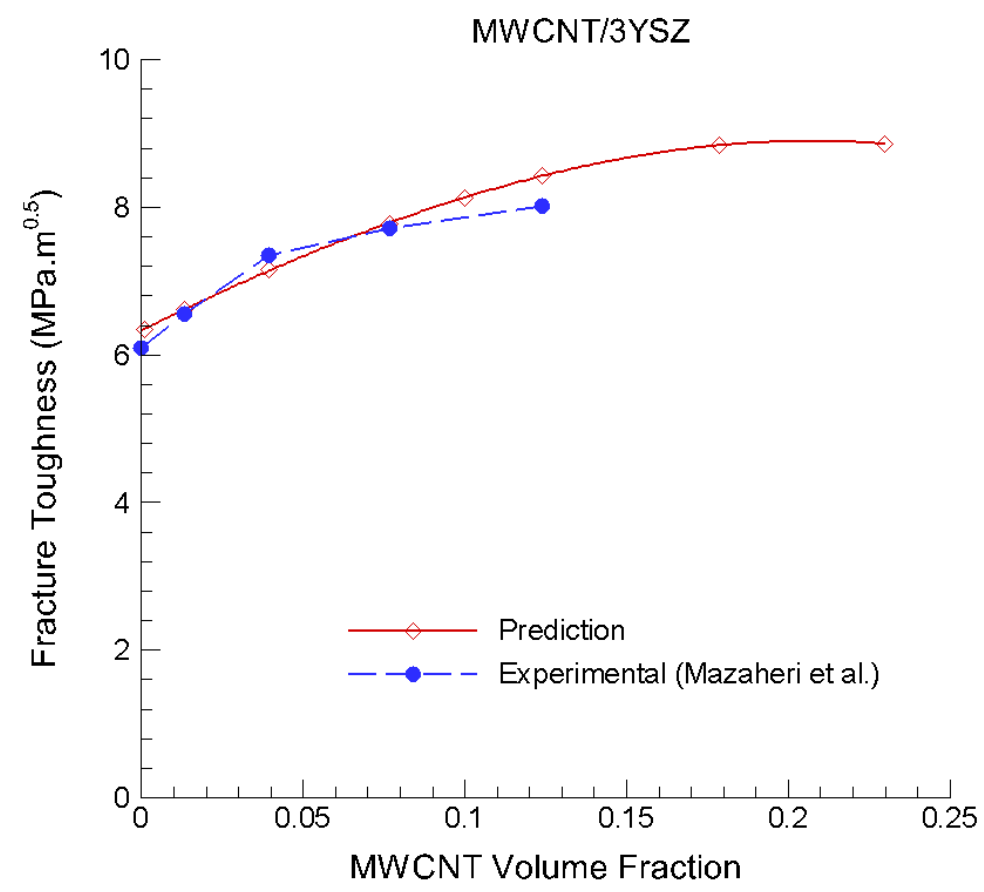

495 Fig. 10. Predicted and experimental Mode I fracture toughness vs. MWCNT volume fraction for 496 the MWCNT/3YSZ composites. The experimental results were determined by Mazaheri et al. [7-8] via bending tests of single-edge V-notched beam specimens. 


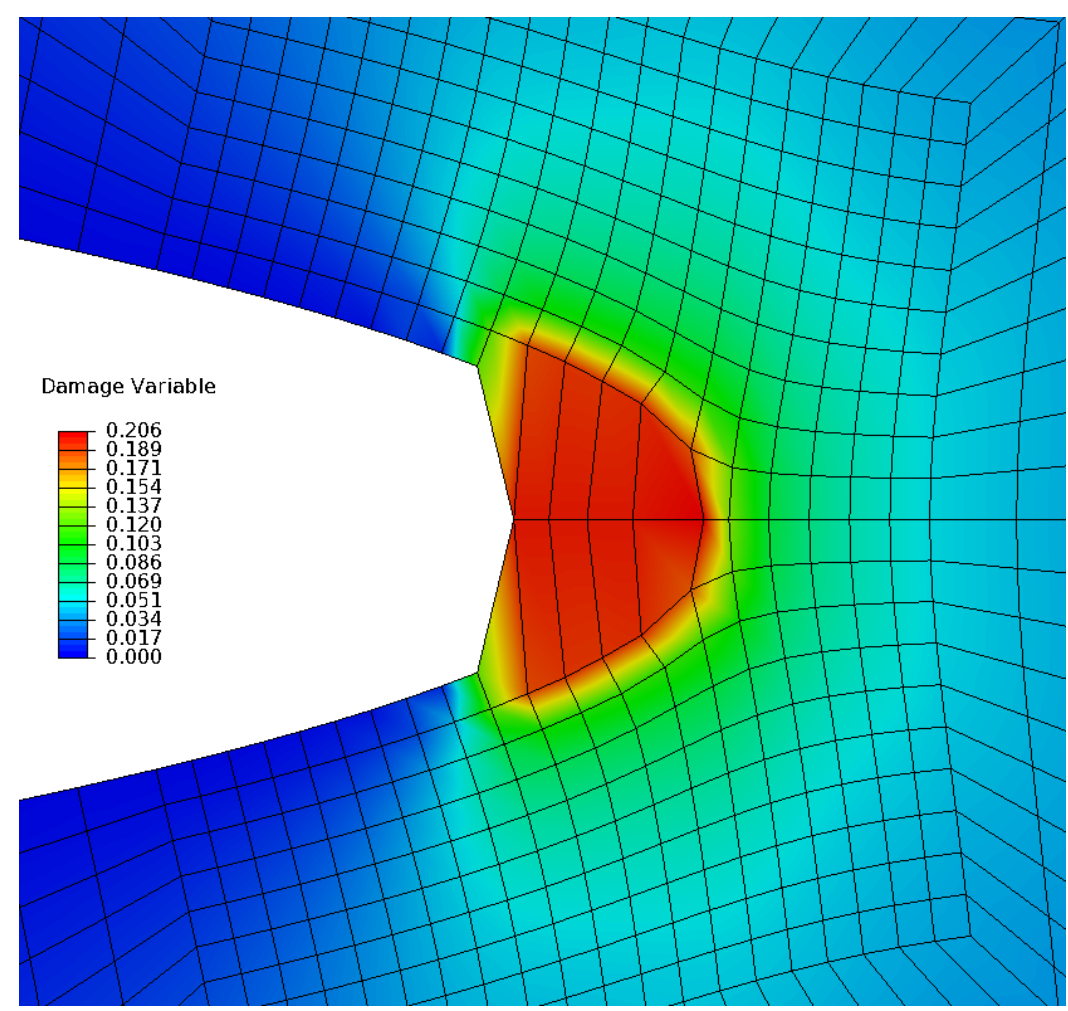

Fig. 11. Development of damage at the loading level $K_{\mathrm{I}}^{\mathrm{R}}=9.11 \mathrm{MPa} \sqrt{\mathrm{m}}$ for the 3 -wt $\%$ MWCNT/3YSZ composite.

Damage can progress further in the process window after initiation if loading is increased. Figure 11 shows damage progression at a higher loading level $K_{I}=9.11 \mathrm{MPa} \mathrm{V}_{\mathrm{m}}$ for the 3 -wt $\%$ 505 MWCNT/3YSZ composite. The present MBL analysis is also used to predict the stress intensity 506 factor vs. crack advance (R-curve) as a function of the MWCNT content. Once the damage 507 variable has attained the critical value at a given integration point in a finite element, the 508 vanishing element method reduces the local element stiffness and stresses to zero in a small 509 number of loading steps, and the stresses are redistributed to the adjacent integration points until 510 the element is completely failed, so that the failed elements represent an open crack. Figure 12 511 illustrates crack propagation in a process window for the 3-wt\% MWCNT/3YSZ composite. The 
512 corresponding stress intensity and crack advance (measured from the initial crack tip) are $5139.49 \mathrm{MPa} \sqrt{\mathrm{m}}$ and $0.245 \mathrm{~mm}$, respectively. The symmetries of the loading and of the finite 514 element mesh with respect to the initial crack plane combined with uniform material properties 515 lead to the symmetric crack propagation shown in Fig. 12. If irregular and asymmetric mesh 516 design or statistical distributions of material parameters were used for the process window, an 517 asymmetric crack pattern would have occurred. The crack propagation in that case would 518 represent a lower amount of work and thus a lower stress intensity factor to drive the crack by a 519 specific length. Thus, the symmetric crack propagation shown here is overly conservative.

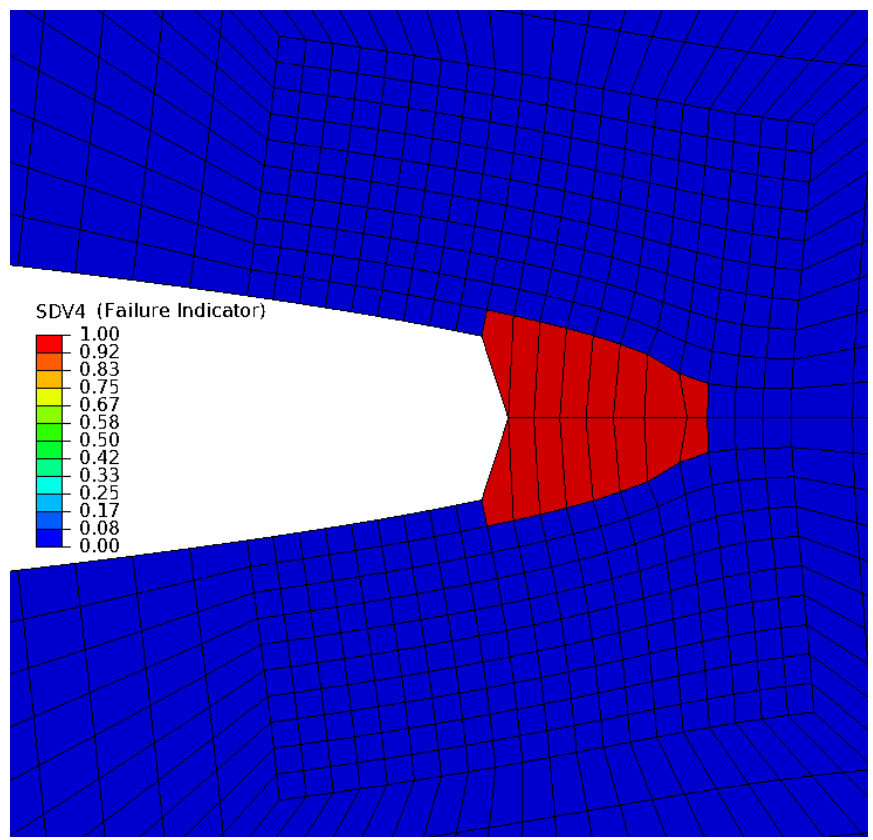

521 Fig. 12. Predicted crack propagation for the $3-\mathrm{wt} \% \mathrm{MWCNT} / 3 \mathrm{YSZ}$ at $K_{\mathrm{I}}=K_{\mathrm{I}}^{\mathrm{R}}=9.47 \mathrm{MPa} \sqrt{\mathrm{m}}$.

The R-curves also predicted by the MBL analysis for all other MWCNT/3YSZ composites with weight fractions from 0.04 to $5 \%$ and the results are reported in Fig. 13. For the extent of 525 the crack advance predicted, all the predicted R-curves can be fitted quite well by a power-law of 526 the form: 
528 where $K_{\mathrm{I}}^{\mathrm{R}}$ is stress intensity factor after the onset of crack propagation, and $c$ is the crack 529 advance, and $k$ and $\alpha$ are fitting coefficients. Using Eq. 17, there exists an initial value of $c$ $530 \quad\left(c=c_{0}\right)$ for which $K_{\mathrm{I}}^{\mathrm{R}}=k c_{0}^{\alpha}=K_{\mathrm{I}}^{\mathrm{c}}$, the fracture toughness. Although the experimental R-curves 531 for the MWCNT/3YSZ composites studied in this paper are not known, the predicted power-law 532 trend (Eq. 17) was also experimentally observed in other ceramic composite systems [46-47]. 533 Table 1 gives the values of $k$ and $\alpha$ obtained from the fitting of the MBL analysis results (Fig. 534 13) by the power-law in Eq. 17.

\section{MWCNT/3YSZ}

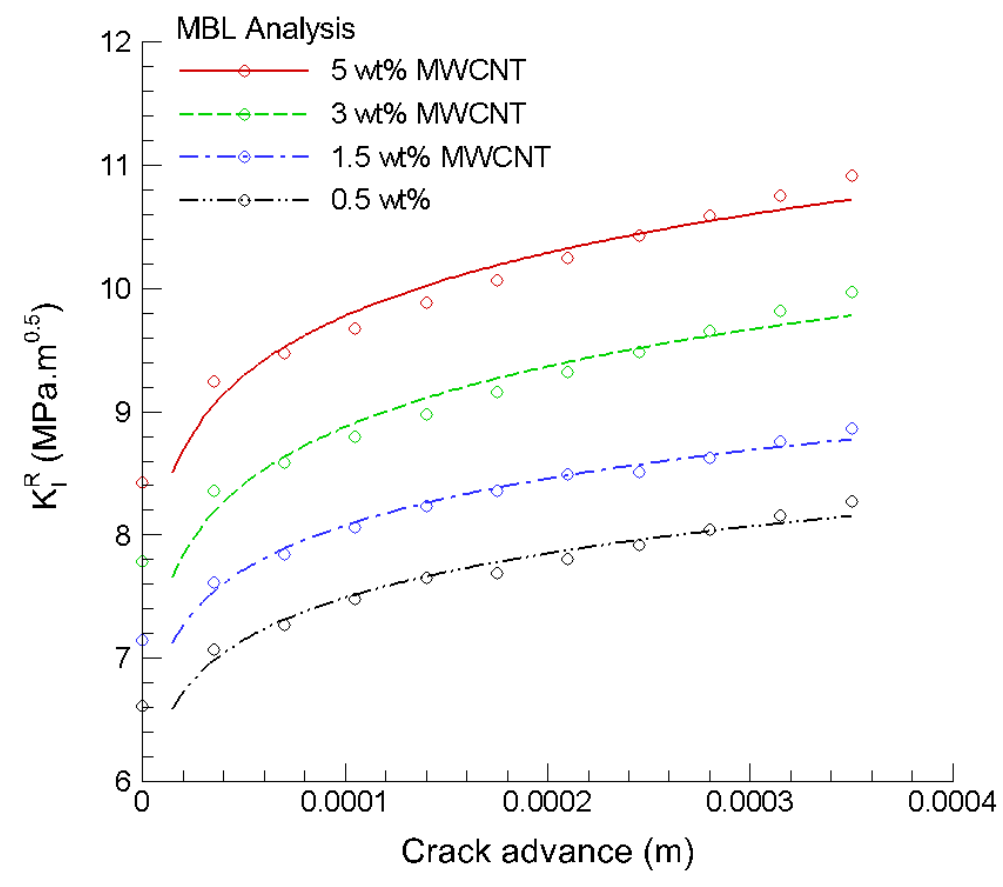

536 Fig. 13. Predicted stress intensity factor vs. crack advance for the MWCNT/3YSZ composites as 537 a function of MWCNT content. As discussed above, these computed R-curves represent symmetric crack advance and would be expected to be somewhat lower for other crack advance assumptions. 
Table 1. R-curves power-law coefficients identified for the MWCNT/3YSZ composites studied in this paper.

\begin{tabular}{|c|c|c|c|c|}
\hline MWCNT Content & $\mathbf{0 . 0 5}-w \mathbf{~} \%$ & $\mathbf{1 . 5}-\mathbf{w t} \%$ & 3-wt \% & 5-wt \% \\
\hline$c_{0}(\mathrm{~m})$ & $1.5 \times 10^{-5}$ & $1.5 \times 10^{-5}$ & $1.5 \times 10^{-5}$ & $1.5 \times 10^{-5}$ \\
\hline$k\left(\mathrm{MPa} \cdot \mathrm{m}^{1 / 2} \cdot \mathrm{m}^{-\alpha}\right)$ & 13.9 & 14.8 & 18.1 & 19.2 \\
\hline$\alpha$ & $6.7 \times 10^{-2}$ & $6.6 \times 10^{-2}$ & $7.7 \times 10^{-2}$ & $7.3 \times 10^{-2}$ \\
\hline
\end{tabular}

\section{Discussion of Results}

The developed damage model, when compared to experimental data, appears to accurately predict the observed results and, since the model is quite general in scope, suggests that CNT strength saturation and decline is to be expected for these materials and that an optimum volume fraction of CNTs exists for a given ceramic matrix depending on the manner in which the matrix undergoes elastic damage in the presence of distributed CNTs.

To understand strength saturation, the evolution of the damage variable with applied stress on the composite is plotted as a function of CNT volume fraction in Fig. 14. These curves show that matrix damage increases with applied stress as expected but damage increases are accompanied by increased load carrying capability for CNT volume fractions in the range from 0 to $23 \%$. Increasing CNT volume fraction in this range augments the load carrying capacity of the composite for the same applied stress since stresses are distributed to more CNTs, and as a result, this reduces stress in the matrix leading to lower matrix damage accumulation. However, at higher CNT fractions beyond $23 \%$, for the same applied stress, more stresses redistributed to the matrix cause higher matrix stresses and strains leading to more matrix damage. Fig. 14 shows clearly that at $40 \%$ volume fraction, the damage variable rapidly increases with the applied stress 
559 to the critical value $\left(D_{\mathrm{cr}}=0.2\right.$ ), which is the same as the unreinforced ceramic matrix but at 560 much lower applied strains, engendering early failure of the composite (see Fig. 6).

561 Another way to help understand reductions of composite strength and failure strain at high

562 CNT volume fractions is to examine the computed stresses on the ceramic matrix as a function of 563 CNT fraction for given levels of applied strain as shown in Fig. 15, which shows the evolution of 564 the matrix stress $\sigma_{11}^{\mathrm{m}}$ with CNT fraction for different values of the composite strain $\varepsilon_{11}$. For $565 \varepsilon_{11}=0.001$ and CNT volume fractions less than $23 \%, \sigma_{11}^{\mathrm{m}}$ increases little with increasing CNT 566 fraction, but it evolves substantially for volume fractions beyond $23 \%$, and at about $40 \%$ CNT 567 volume fraction the matrix stress $\sigma_{11}^{\mathrm{m}}$ has almost attained the matrix tensile failure stress of 713 $568 \mathrm{MPa}$ (see Fig. 4). Higher composite strain levels induce higher matrix stresses as indicated in 569 Fig. 15, which also shows that composites with CNT fractions less than about $13 \%$ can sustain 570 an applied strain four times the value the composite at $40 \%$ can support. The applied stress 571 distribution to the ceramic matrix is nonlinear with respect to CNT volume fraction, which 572 cannot be intuited from Eq. 12. 
573

\section{4}

575

576

577 the matrix strain $\varepsilon_{11}^{\mathrm{m}}$ as a function of the composite stress $\sigma_{11}$ (in the loading direction) and CNT

578 volume fraction. In the range of volume fractions less than $23 \%$, increasing the CNT volume

579 fraction reduces the matrix strain for the same composite stress, but for volume fractions higher

580 than $23 \%$, an increase in volume fraction at the same composite stress causes an increase in

581 matrix strain. As the matrix strains govern the damage evolution law (Eq. 12), an increase in the

582 matrix strain produces an increase in the damage variable increment accordingly, and therefore

583 greater damage accumulation. In a practical sense, addition of CNTs to ceramics increases the

584 overall stiffness of the resultant composite at the expense of failure strain and matrix load 585 carrying capability. 


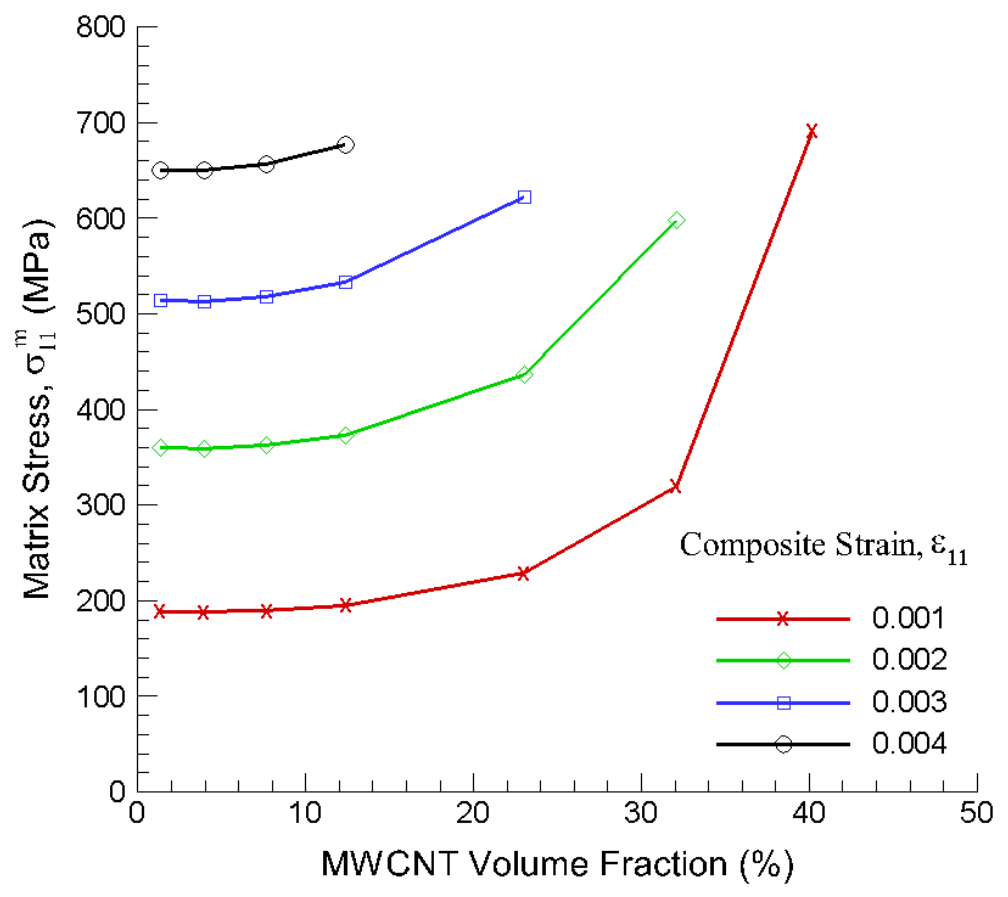

587 Fig. 15. Evolution of the matrix stress $\sigma_{11}^{\mathrm{m}}$ with MWCNT volume fraction for different levels of composite strain $\varepsilon_{11}$.

589 The composite stress/strain results reported in Fig. 6 present one of the most important 590 findings in this work. They show that even under the ideal conditions of well-dispersed CNTs 591 and perfect bonding, the composite strength reaches a limit beyond which adding more 592 reinforcements will not lead to improved composite strength and failure strain, but rather serve to 593 decrease failure strain beyond a certain limit that is likely governed by the modulus ratio of the 594 MWCNTs to the ceramic matrix. It is significant that such reductions and limits in strength and 595 failure strain with the concentration of nano-reinforcements have been also observed 596 experimentally in MWCNT/epoxy [48] and silica nanoparticle reinforced epoxy composites [49]. 


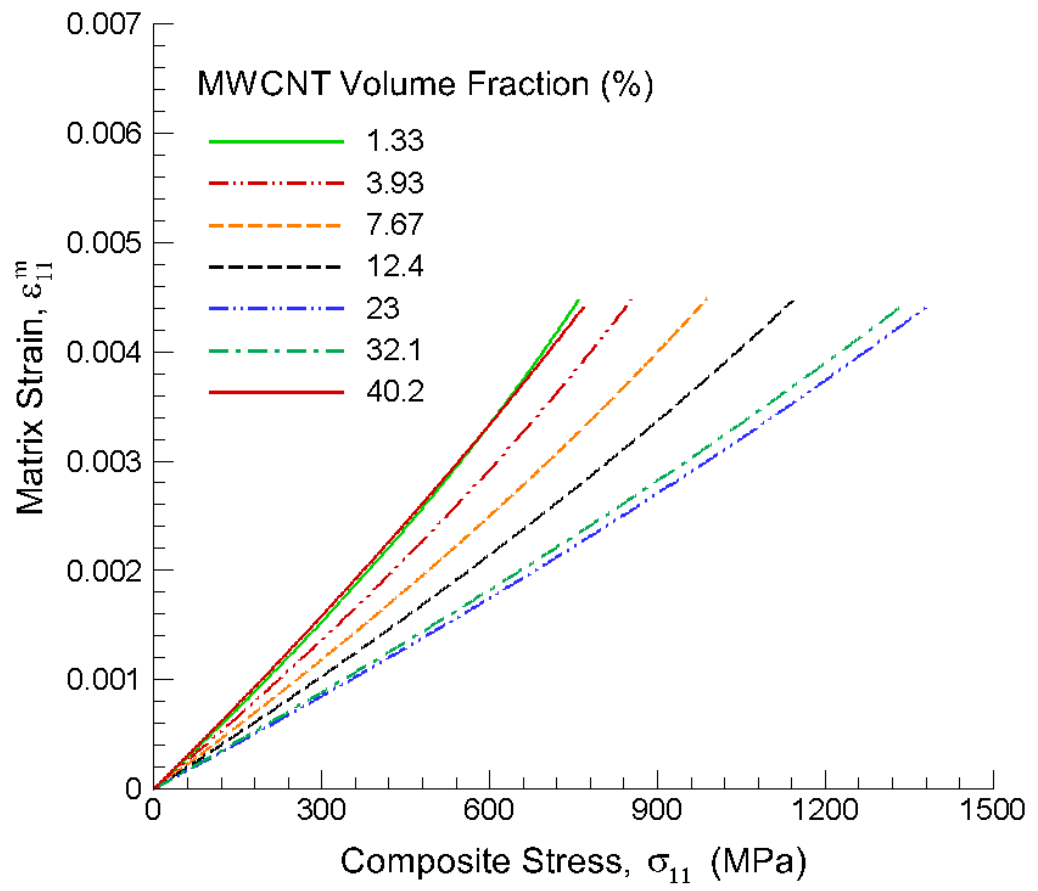

598 Fig. 16. Evolution of the matrix strain $\varepsilon_{11}^{\mathrm{m}}$ as a function of the composite stress $\sigma_{11}$ and MWCNT volume fraction. Similarly, the fracture toughness calculations show the same trends, and the crack 601 propagation model presented here predicts a modest increase in fracture toughness with 602 increasing CNT fraction, followed by a saturation level and then a decrease. Saturation is 603 predicted to occur at about the same level, 20 to $25 \%$, at which the strength degradation occurs in 604 the damage model (see Figs. 6 and 13). Although the damage model here does not discretely 605 capture fiber pullout, the model does capture the final stage of damage by numerically reducing 606 the stresses and stiffness at the material failure location to zero in a number of load steps. When 607 data for 3YSZ/MWCNT composites is used the model agreement is excellent as shown in Fig. 608 13. As significant as Fig. 6, the results from Fig. 13 are also significant and suggest that only 609 modest toughness increases can be achieved by dispersed CNT reinforcements, and for the same 
610

611

612

613

614

615

616

617

618

619

620

621

622

623

624 625 significant increases in the mechanical properties of the as-formed composite, which has a 3D

626

627

628

629

630

631 632 fracture toughness values.

A modeling approach to predict the fracture toughness and crack resistance behavior of CNT-reinforced ceramic composites is developed and described to provide insight and guidance to processing this class of composite materials to determine if it was possible to achieve the levels of fracture toughness required for applications in advanced nuclear reactors, for example. A series of computational steps including computations of the CNT composite elastic properties and nonlinear stress/strain responses due to micromechanical damage was performed prior to the prediction of fracture toughness. Models for elastic properties and damage by matrix cracking are developed and implemented in predictive tools. The damage model associated with a modified boundary layer modeling approach is able to efficiently predict composite toughness and crack resistance behavior. Predictions of elastic properties and fracture toughness agree well with experimental results from MWCNT/3YSZ composites. The modeling results show that homogeneously dispersing low to moderate contents of CNTs in a ceramic matrix leads to CNT orientation distribution. However, the increase in strength and toughness saturates at high CNT contents, which is likely due to the presence of the much stiffer CNT reinforcement. Therefore, it is not possible to achieve the levels of fracture toughness, estimated to be $K_{\mathrm{I}}^{\mathrm{c}} \geq 20 \mathrm{MPa} \sqrt{\mathrm{m}}$, considered to be needed for nuclear reactor applications using distributed CNTs in typical ceramic matrices. Currently, remedying this limitation is being addressed by studying other composite microstructures and architectures designed to achieve higher Mode I 
634 This research was funded by the United States Department of Energy (US DOE) Office of 635 Nuclear Energy under the Nuclear Energy Enabling Technology Reactor Materials (NEET-RM) 636 Program. The US DOE Office of Vehicle Technologies funded initial developments of EMTA 637 and EMTA-NLA software tools. PNNL is a multi-program national laboratory operated by 638 Battelle Memorial Institute for the US Department of Energy under DE-AC06-76RLO 1830.

639

640

$641 \quad$ Mater Sci. 1994;29:3857-96.

642 [2] Ruehle M, Evans AG. High toughness ceramics and ceramic composites. Prog Mater Sci. $643 \quad 1989 ; 33: 85-167$.

644 [3] Taheri M, Mazaheri M, Golestani-fard F, Rezaie H, Schaller R. High/room temperature 645 646 647 648 649 review. Int Mater Rev. 2010;55:41-64.

650 [6] Jian Y, Ting W, Zhipeng X, Weijiang X. Zirconia-based nanocomposite toughened by 651 functionalized multi-wall carbon nanotubes. J Alloys Compd. 2013;581:452-8. 
652 [7] Mazaheri M, Mari D, Schaller R, Bonnefont G, Fantozzi G. Processing of yttria stabilized 653 zirconia reinforced with multi-walled carbon nanotubes with attractive mechanical properties. J

654 Eur Ceram Soc. 2011;31:2691-8.

655 [8] Mazaheri M, Mari D, Hesabi ZR, Schaller R, Fantozzi G. Multi-walled carbon 656 nanotube/nanostructured zirconia composites: Outstanding mechanical properties in a wide range 657 of temperature. Compos Sci Technol. 2011;71:939-45.

658 [9] Kuskonmaz N, Ozturk ZT. High pressure sintering of Al2O3-CNT nanocomposites. Mech 659 Testing. 2014;56:479-82.

660 [10] Gu Z, Yang Y, Li K, Tao X, Eres G, Howe JY, et al. Aligned carbon nanotube-reinforced 661 silicon carbide composites produced by chemical vapor infiltration. Carbon. 2011;49:2475-82.

662 [11] Balazsi C, Tapaszto O, Karoly Z, Kun P, Balazsi K, Szepvolgyi J. Structural and 663 mechanical properties of milled $\mathrm{Si}_{3} \mathrm{~N}_{4} / \mathrm{CNT}$ composites by spark plasma sintering method. 664 Mater Sci Forum. 2013;729:31-6.

665 [12] Poyato R, Gallardo-Lopez A, Gutierrez-Mora F, Morales-Rodriguez A, Munoz A, 666 Dominguez-Rodriguez A. Effect of high SWNT content on the room temperature mechanical 667 properties of fully dense 3YTZP/SWNT composites. J Eur Ceram Soc. 2014;34:1571-9.

668 [13] Xia Z, Riester L, Curtin WA, Li H, Sheldon BW, Liang J, et al. Direct observation of 669 toughening mechanisms in carbon nanotube ceramic matrix composites. Acta Mater. $670 \quad 2004 ; 52: 931-44$.

671 [14] Desai AV, Haque MA. Mechanics of the interface for carbon nanotube-polymer composites. 672 Thin-Walled Structures. 2005;43:1787-803. 
673 [15] Mori T, Tanaka K. Average stress in matrix and average elastic energy of materials with 674 misfitting inclusions. Acta Metall. 1973;21:571-4.

675 [16] Eshelby JD. The elastic field outside an ellipsoidal inclusion. Proceedings of the Royal 676 Society of London, Series A (Mathematical and Physical Sciences). 1959;252:561-9.

677 [17] Benveniste Y. A new approach to the application of Mori-Tanaka's theory in composite 678 materials. Mech Mater. 1987;6:147-57.

679 [18] Thostenson ET, Li C, Chou T-W. Nanocomposites in context. Compos Sci Technol. $680 \quad 2005 ; 65: 491-516$.

681 [19] Lu WB, Wu J, Song J, Hwang KC, Jiang LY, Huang Y. A cohesive law for interfaces 682 between multi-wall carbon nanotubes and polymers due to the van der Waals interactions. Comp 683 Meth Appl Mech Engr. 2008;197:3261-7.

684 [20] Odegard GM, Gates TS, Wise KE, Park C, Siochi EJ. Constitutive modeling of nanotube685 reinforced polymer composites. Composites Sci Technol. 2003;63:1671-87.

686 [21] Odegard GM, Gates TS, Nicholson LM, Wise KE. Equivalent-continuum modeling of nano687 structured materials. Composites Sci Technol. 2002;62:1869-80.

688 [22] Frankland SJV, Harik VM, Odegard GM, Brenner DW, Gates TS. The stress-strain behavior 689 of polymer-nanotube composites from molecular dynamics simulation. Composites Sci Technol. $690 \quad 2003 ; 63: 1655-61$.

691 [23] Fisher FT, Bradshaw RD, Brinson LC. Fiber waviness in nanotube-reinforced polymer 692 composites-I: Modulus predictions using effective nanotube properties. Composites Sci Technol. $693 \quad 2003 ; 63: 1689-703$. 
694 [24] Bradshaw RD, Fisher FT, Brinson LC. Fiber waviness in nanotube-reinforced polymer 695 composites-II: Modeling via numerical approximation of the dilute strain concentration tensor. 696 Compos Sci Technol. 2003;63:1705-22.

697 [25] Shi D-L, Feng X-Q, Huang YY, Hwang K-C, Gao H. The effect of nanotube waviness and 698 agglomeration on the elastic property of carbon nanotube-reinforced composites. J Engr Mater 699 Technol, Trans ASME. 2004;126:250-7.

700 [26] Shao LH, Luo RY, Bai SL, Wang J. Prediction of effective moduli of carbon nanotube701 reinforced composites with waviness and debonding. Composite Struct. 2009;87:274-81.

702 [27] Li C, Chou T-W. Failure of carbon nanotube/polymer composites and the effect of nanotube 703 waviness. Composites Part A: Applied Science and Manufacturing. 2009;40:1580-6.

704 [28] Seidel GD, Lagoudas DC. Micromechanical analysis of the effective elastic properties of 705 carbon nanotube reinforced composites. Mech Mater. 2006;38:884-907.

706 [29] Hashin Z, Rosen BW. The Elastic Moduli of Fiber-Reinforced Materials. J Appl Mech. $707 \quad 1964 ; 31: 223-32$.

708 [30] Schadler LS, Giannaris SC, Ajayan PM. Load transfer in carbon nanotube epoxy 709 composites. Appl Phys Lett. 1998;73:3842-4.

710 [31] Barai P, Weng GJ. A theory of plasticity for carbon nanotube reinforced composites. Int J 711 Plast. 2011;27:539-59.

712 [32] Dong S, Zhou J, Hui D, Wang Y, Zhang S. Size dependent strengthening mechanisms in 713 carbon nanotube reinforced metal matrix composites. Composites A: Appl Sci Manuf. $714 \quad 2015 ; 68: 356-64$. 
715 [33] Tvergaard V, Hutchinson JW. The relation between crack growth resistance and fracture 716 process parameters in elastic-plastic solids. J Mech Phys Solids. 1992;40:1377-97.

717 [34] Nguyen B-N, Onck P, van der Giessen E. Crack-tip constraint effects on creep fracture. Eng 718 Fract Mech. 2000;65:467-90.

719 [35] Nguyen BN, Koeppel BJ, Ahzi S, Khaleel MA, Singh P. Crack Growth in Solid Oxide Fuel 720 Cell Materials: From Discrete to Continuum Damage Modeling. J Am Ceram Soc. $721 \quad 2006 ; 89: 1358-68$.

722 [36] Mura T. Micromechanics of Defects in Solids. Second Revised ed. Boston, MA: Kluwer 723 Academic Publishers; 1987.

724 [37] Advani SG, Tucker Iii CL. The Use of Tensors to Describe and Predict Fiber Orientation in 725 Short Fiber Composites. J Rheol. 1987;31:751-84.

726 [38] Wetzel ED. Modeling flow-induced microstructure of inhomogeneous liquid-liquid 727 mixtures [PhD Thesis]. Urbana, IL: University of Illinois Urbana-Champaign; 1999.

728 [39] Mukhopadhyay A, Chu BTT, Green MLH, Todd RI. Understanding the mechanical 729 reinforcement of uniformly dispersed multiwalled carbon nanotubes in alumino-borosilicate 730 glass ceramic. Acta Mater. 2010;58:2685-97.

731 [40] Maugin GA. The Thermomechanics of Plasticity and Fracture. Cambridge, UK: Cambridge 732 University Press; 1992.

733 [41] Renard J, Favre JP, Jeggy T. Influence of transverse cracking on ply behavior: introduction 734 of a characteristic damage variable. Compos Sci Technol. 1993;46:29-37. 
735 [42] Lemaitre J. Introduction to Continuum Damage Mechanics. In: Allix O, Hild F, editors. 736 Continuum Damage Mechanics of Materials and Structures. Oxford, UK: Elsevier Science Ltd; 737 2002. p. 235-58.

738 [43] Tvergaard V. Analysis of creep crack growth by grain boundary cavitation. Int J Fract. 739 1986;31:183-209.

740 [44] Kondoh J, Shiota H, Kawachi K, Nakatani T. Yttria concentration dependence of tensile 741 strength in yttria-stabilized zirconia. J Alloys Compounds. 2004;365:253-8.

742 [45] Onck P, van der Giessen E. Growth of an initially sharp crack by grain boundary cavitation. $743 \quad J$ Mech Phys Solids. 1998;47:99-139.

744 [46] Ramachandran N, Shetty DK. Rising crack-growth-resistance (R-curve) behavior of 745 toughened alumina and silicon nitride. J Am Ceram Soc. 1991;74:2634-41.[47] Sarkar S, Das 746 PK. Temperature and load dependent mechanical properties of pressureless sintered carbon 747 nanotube/alumina nanocomposites. Mater Sci Engr A. 2012;531:61-9.

748 [48] Ogasawara T, Moon S-Y, Inoue Y, Shimamura Y. Mechanical properties of aligned 749 multiwalled carbon nanotube/epoxy composites processed using a hot-melt prepreg method. 750 Compos Sci Technol. 2011;71:1826-1833.

751 [49] Jumahat A, Soutis C, Abdullah SA, Kasolang S. Tensile Properties of Nanosilica/Epoxy 752 Nanocomposites. Proc Engr. 2012;41:1634-40. 